Running Head: CULTURE AND PUNITIVE BELIEF

Tight Cultures and Vengeful Gods: How Culture Shapes Religious Belief

Joshua Conrad Jackson ${ }^{1 *}$, Nava Caluori², Samantha Abrams ${ }^{1}$, Elizabeth Beckman ${ }^{3}$, Michele Gelfand $^{4}, \&$ Kurt Gray ${ }^{1}$

1. University of North Carolina at Chapel Hill, Department of Psychology and Neuroscience

2. University of Virginia, Department of Psychology

3. University of North Carolina at Chapel Hill, Department of Philosophy

4. University of Maryland, Department of Psychology

*Corresponding Author

Department of Psychology

University of North Carolina, Chapel Hill

Chapel Hill, NC 27599

Email: joshcj@live.unc.edu

Phone: 202-821-7436 


\begin{abstract}
Billions of people from around the world believe in vengeful gods who punish immoral behavior. These punitive religious beliefs may foster prosociality and contribute to large-scale cooperation, but little is known about how these beliefs emerge and why people adopt them in the first place. We present a cultural-psychological model suggesting that cultural tightness - the strictness of cultural norms and normative punishment- helps to catalyze punitive religious beliefs by increasing people's motivation to punish norm violators. Our model also suggests that tightness mediates the impact of ecological threat on punitive belief, explaining why punitive religious beliefs are most common in regions with high levels of ecological threat. Five multimethod studies support these predictions. Studies 1-3 focus on the effect of cultural tightness on punitive religious beliefs. Historical increases in cultural tightness precede and predict historical increases in punitive beliefs (Study 1), and both manipulating people's support for tightness (Study 2) and placing people in a simulated tight society (Study 3 ) increase punitive religious beliefs via the personal motivation to punish norm violators. Studies 4-5 focus on whether cultural tightness mediates the link between ecological threat and punitive religious beliefs. Cultural tightness helps explain why U.S. states with high ecological threat (e.g. natural hazards, scarcity) have the highest levels of punitive religious beliefs (Study 4), and why experimental manipulations of threat increase punitive religious beliefs (Study 5). Past research shows how religion impacts culture, but our studies show how culture can shape religion.
\end{abstract}

Keywords: Cultural Psychology; Religious Belief; Cultural Evolution; New Methods 


\section{Tight Cultures and Vengeful Gods: How Culture Shapes Religious Belief}

Supernatural punishment is a defining feature of many modern-day religions. From the punitive God of Christianity and Islam, to the retributive principle of Karma in Hinduism and Buddhism, to posthumous landscapes of pain in many religions, billions of people believe that divine damnation awaits those who violate earthly edicts. These punitive religious beliefs may be effective for promoting and sustaining large-scale cooperation (Norenzayan \& Shariff, 2008), but there are many open questions about the origins and transmission of these beliefs, especially because they vary considerably across cultures (Hackett et al., 2012). Past research suggests that several forms of ecological threat are linked with punitive religious beliefs (Botero et al., 2014), but only rarely do studies establish mechanisms and causal directions for these links. As a result, there is still no widely accepted account of how punitive religious beliefs emerge and spread over time, despite the frequent discussion of these beliefs in psychology (Norenzayan et al., 2016), anthropology (Johnson, 2015), and history (Slingerland et al., 2020).

The goal of this paper is to outline a cultural-psychological model of punitive religious beliefs: Cultural tightness - the strictness of societal norms - encourages punitive religious beliefs because it increases people's motivation to punish norm violators, and punitive religious beliefs help to fulfill this motivation. Because cultural tightness is especially adaptive in the face of ecological threat (Roos et al., 2015), we further predict that cultural tightness can explain why punitive religious beliefs are most prevalent in regions with high levels of threat. We call this a cultural-psychological model because it offers a multi-level framework for understanding how culture-level variation in societal (cultural tightness) and environmental factors (ecological threat) can influence individual-level psychological processes (the motivation to punish norm violators), ultimately catalyzing changes in the nature of religious beliefs. While some studies 
have suggested that cultural tightness may explain why conflict can change religious belief (Caluori, 2020), our research program is the first to show that cultural tightness is associated with the historical and geographic distribution of punitive religious beliefs, and can explain why a broad range of ecological threats are linked to more moralizing and punitive belief systems.

We test these predictions with an array of different methods. Through a historical textual analysis using time-series methods, we show that historical variation in cultural tightness can predict time-lagged shifts in people's tendency to quote Bible chapters with punitive themes. Through a cross-cultural survey of religion across the United States, we use path modeling to show that cultural tightness can help explain why distal ecological threats predict the modern distribution of punitive religious beliefs. And with three experiments, we show that cultural tightness increases punitive religious beliefs, mediates the effect of ecological threat on punitive religious beliefs, and that there is evidence for a serial mediation from ecological threat to cultural tightness to the motivation to punish norm violators to punitive religious beliefs.

\section{The Cultural Evolution of Punitive Religious Beliefs}

Supernatural beliefs may be as ancient as Homo sapiens themselves (Fogelin, 2007), but believing in spirits and gods that punish humans for immoral behavior may be more novel. Historical cross-cultural surveys suggest that beliefs in some forms of supernatural punishment may precede the Neolithic revolution (Watts et al., 2015), but these supernatural punishments could be idiosyncratic and unrelated to human morality. In the Sumerian Atrahasis epic, for example, the gods flood the earth simply because humans were disturbing their rest. Beliefs in gods that punish humans for moral and normative violations (the focus of our paper) appear to be

much more recent (Peoples, Duda, \& Marlowe, 2016; Watts et al., 2015), and may have emerged as early as 4,000 years ago (Whitehouse et al., 2019). The exact date of this emergence is 
difficult to truly determine because many societies did not have written records until recent human history (Beheim et al., 2019), but most scholars agree that punitive religious beliefs emerged at some point during the Holocene epoch $\left(\sim 12,000 \mathrm{cal} \_\right.$present $)$and then spread to many societies (Johnson, 2016; Norenzayan et al., 2016).

In the past 20 years, research on the emergence, spread, and impact of punitive beliefs has become a major area of interest, with prominent books and papers accumulating thousands of citations and drawing interest across the social sciences (Bulbulia, 2004; Johnson, 2016; Norenzayan, 2013). Most of this past research has been grounded in the framework of cultural evolution, which suggests that culture follows systematic patterns of transmission and inheritance that mimic some (but not all) forms of Darwinian evolution (Brewer et al., 2017). Cultural evolution suggests that cultural traits can be inherited across generations, that this inheritance process can include modifications to cultural beliefs and practices that resemble mutation, and that cultures' local ecologies can have a powerful impact on this process of inheritance (Gray \& Watts, 2019; Mesoudi, Whiten, \& Laland, 2006).

There is an important distinction in cultural evolution between distal and proximal explanations for the emergence of traits (Brewer et al., 2017; Tinbergen, 1963). Distal explanations focus on how cultural traits evolve because they are adaptive for human groups, whereas proximal explanations focus on how cultural traits evolve because they are appealing to individual people within a cultural group (Sperber, 1996). For example, a distal explanation of the adoption of spices in cooking is that it has antimicrobial functions, but a proximal explanation could be that spices taste good (Billing \& Sherman, 1998).

Most theories of punitive religion focus on distal explanation, arguing that punitive religious beliefs evolve because they promote large-scale cooperation, which is necessary to 
sustain complex societies. In support of these models, many studies find that priming gods and karma increases parochial cooperation (Shariff, Willard, Anderson, \& Norenzayan, 2016; White, Kelly, Shariff, \& Norenzayan, 2020), that religious priming is most effective at promoting prosocial intentions when it involves punitive aspects of God (Yilmaz \& Bahcekapili, 2016), and that groups with punitive religious beliefs are more prosocial than groups without punitive religious beliefs (Johnson, 2005; Johnson \& Krüger, 2004; Purzycki et al., 2016; Shariff \& Rhemtulla, 2012). These effects suggest that punitive religious beliefs might be adaptive for human groups because large-scale cooperation is necessary for taxation systems, military recruitment, market economies, and many other aspects of complex societies (Murdock \& Provost, 1973). The communal benefits of punitive religious beliefs imply that - once established — these beliefs should be persistent.

However, this distal explanation of punitive religious beliefs does not explain why individuals are inclined to embrace and share these beliefs, especially because increasing grouplevel cooperation can undermine the outcomes of individuals, at least in the near term. Giving away your money to an anonymous stranger may foster a more charitable society, but it also makes you a poorer person. Why, then, do people adopt punitive religious beliefs?

Answering the proximal question of "why" people might adopt punitive religious beliefs might also help answer the question of "where" these beliefs should culturally evolve. There is considerable historical and geographic variation in punitive religious beliefs (Purzycki et al., 2016), but the sources of this variation are not clear. Even in nations like the USA, which have generally high levels of punitive religious beliefs, some regions endorse these beliefs more than others (Hackett et al., 2012). A recent high-profile analysis suggested that punitive beliefs emerge after societies grow and become more complex (Whitehouse et al., 2019; c.f. Beheim et 
al., 2020), but the United States is fully industrialized and every U.S. state has high social complexity and yet there is still high variability in punitive religious beliefs across the country (Hackett et al., 2012).

Another line of evidence suggests that punitive religious beliefs are most common in the face of high environmental distress (Botero et al., 2014) and warfare (Caluori et al., 2020). At first glance, the existence of environmental distress and warfare would seem to have little connection to punitive religious beliefs, but it is exactly these elements that seem to increase the tightness of social norms, and we suggest that tightness may be the crucial information for predicting when and why punitive religious beliefs might be appealing to individuals.

\section{Cultural Tightness May Catalyze Punitive Religious Beliefs}

Scholars since Sima Qian, Herodotus, and Polybius have recognized that societies vary in their strictness of norms and punishment. However, the words "tight" and "loose" were only coined many years later by the anthropologist Pelto (1968), in a paper where he contrasted "tight" societies that had rigid norms and harsh punishments for normative deviance with "loose" groups that had more flexible norms and greater tolerance for norm violators. Pelto gave the Hutterites - a group much like the Amish who live in the American Great Plains - as an example of a prototypically tight group while describing the Sami-reindeer herders in current-day Norway and Sweden - as a loose group.

Building on these descriptive accounts, Triandis (1989) and Gelfand (2011) laid out a more comprehensive theory of cultural tightness's antecedents and consequences. Gelfand (2011) suggested that cultural tightness arises partly as a cultural adaptation to ecological threats such as storms, disease outbreaks, wars, and famines. These threats threaten to destabilize societies of any scale, both because they increase the potential for crime and violence and 
because they deplete institutions of law and order such as police forces and judicial systems. Evolutionary models suggest that people gravitate towards cultural tightness in these times to avoid chaos (Roos et al., 2015), and empirical studies suggest that historical ecological threat predicts tightness in modern countries (Gelfand et al., 2011), non-industrial societies (Jackson, Gelfand, \& Ember, 2020), and American States (Harrington \& Gelfand, 2014).

While tightness may emerge as a group-level adaptation, it has important individual-level consequences for cognition and motivation. On average, people in tight societies show greater conscientiousness (Harrington \& Gelfand, 2014), less creativity (Chua, Roth, \& Lemoine, 2015; Jackson et al., 2019a), more neurobiological sensitivity to norm violators (Mu, Gelfand, Kitayama, \& Han, 2015), and more prejudice towards outsiders than people living in loose societies (Jackson et al., 2019b). People in tight societies are also more likely to support authoritarian leaders who promise to enforce law and order (Atkas et al., 2016; Gelfand, Jackson, \& Harrington, 2016). Even though no studies have directly found that people in tight societies are more motivated to punish norm violators, this past research suggests that cultural tightness may increase people's motivations to punish norm violators, and that people may often outsource this punishment to powerful figures.

We propose that these individual-level effects of tightness provide a proximal explanation of punitive religious beliefs. People in tight societies will not always get to personally punish norm violators, but they can outsource this punishment to gods in much the same way that they outsource punishment to strong leaders. You may not be able to call the police to report a jaywalker, but gods can make sure that jaywalker is judged in the afterlife. You may not be able to make a citizen's arrest on someone who jumps an underground turnstile, but you can pray for their soul to be damned in the eternal underground. By increasing individual 
people's motivation to punish norm violators, cultural tightness may turn frightening and vengeful gods into divine arbiters of justice, catalyzing the emergence of punitive religious beliefs.

No studies have tested this proximal explanation of punitive religious beliefs. However, some research suggests that people do indeed outsource punishment to gods (Laurin, Shariff, Henrich, \& Kay, 2012), and that they are most likely to think of gods are moralizing figures after witnessing injustice. For instance, Purzycki, Stagnaro, and Sasaki (2020) found that people were most likely to attribute moralizing thoughts to gods after experiencing a breach of trust in an economic game, and cross-cultural surveys show that systems of private property-where theft would be a salient concern — are a strong predictor of moralizing religious beliefs (Botero et al., 2014). Other research has shown that, in the Siberian Tyvan Republic, people living in communities with high rates of theft believe that gods are most willing to punish theft (Purzycki, 2013). This descriptive evidence suggests that cultural tightness may increase punitive religious beliefs because it increases people's motivations to punish norm violators.

If cultural tightness is induced by ecological threat, and also catalyzes punitive religious beliefs, this could explain why punitive beliefs are most prevalent in areas with high ecological threat (Botero et al., 2014; Gray \& Wegner, 2010). Societies may develop greater tightness in response to ecological threats to maintain order in society, and this cultural tightness may increase individual people's motivation to punish norm violators and ultimately to adopt more punitive religious beliefs. Caluori and colleagues (2020, Study 3 ) recently provided some evidence for this model by showing that support for stronger norms mediated the relationship between experimentally induced conflict salience and the perceived importance of a more punitive god. Here we build on this early evidence with a broader research program that tests 
whether tightness can explain why many forms of ecological threat are linked with punitive religious beliefs.

\section{Testing Causal Theories with Cross-Cultural and Historical Data}

We predict that cultural tightness increases people's punitive religious beliefs via their motivation to punish norm violators. We also predict that cultural tightness can explain why regions with high ecological threat are the most likely to have punitive religious beliefs (see Figure 1). We test these predictions across five studies, leveraging a suite of methods from social psychology, cultural evolution, and econometrics that balance causal inference with large-scale cross-cultural and historical analysis. Each of these methods is valuable for testing questions of cultural dynamics, and our paper represents one of the first instances where these methods are combined to test an integrated model of cultural evolution. One advantage of combining these methods is that it offers us extremely high power. Taken together, our studies analyze over 55,000 individuals and approximately 1.3 million books across 200 hundred years.

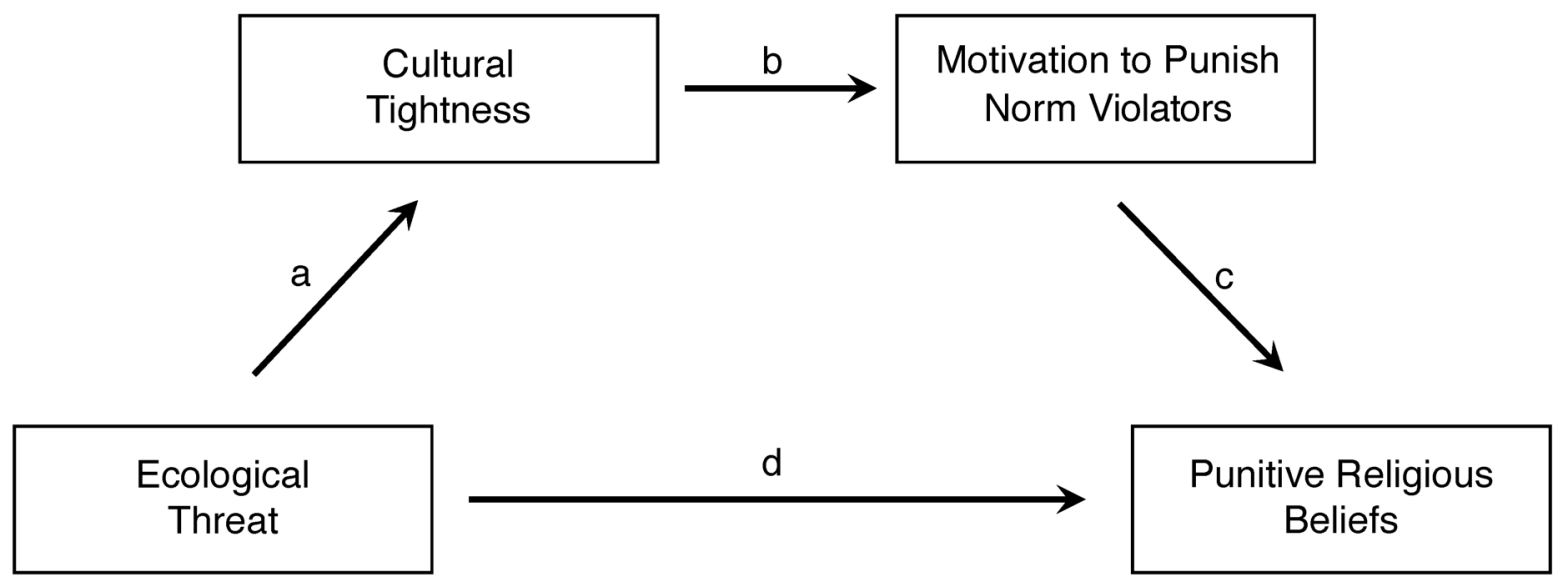

Figure 1. Our theoretical model. We predict that ecological threat increases cultural tightness, which then increases punitive religious beliefs via the motivation to punish norm violators. 
Our first three studies examine the link between cultural tightness and punitive religious beliefs, and explore whether the motivation to punish norm violators is a mechanism that helps explain this link (see Figure 1, paths b \& c). Study 1 examines the relationship between tightness and punitive religious beliefs by analyzing the historical dynamics of punitive religious beliefs. Cultural psychologists have recently applied time-series analyses (traditionally used in econometrics) to predict changes in culture over time (Grossman \& Varnum, 2015; Varnum \& Grossman, 2017; Jackson et al., 2019a), and we leverage these methods in Study 1 to examine whether tightness precedes and predicts punitive religious beliefs from 1800-2000 CE. Studies 23 provide experimental tests of whether cultural tightness increases punitive religious belief. Study 2 manipulates people's favorability towards tight or loose aspects of their society and measures both their motivation to punish norm violators and their punitive religious beliefs. Study 3 conceptually replicates Study 2 by simulating a future society that is either tight or loose and asking participants about their beliefs as a member of this society. We predict in both studies that participants in the tightness condition will show more motivation to punish norm violators, which should mediate the tightness-punitive beliefs relationship.

Our final two studies broaden our model to test whether cultural tightness can explain the relationship between ecological threat and punitive religious beliefs (see Figure 1, paths a-d). Study 4 integrates data on ecological, cultural, and religious variation in the US to test whether cultural tightness can explain geographic variation in punitive religious beliefs across the United States. Through a series of cross-cultural path models, we predict that states with high rates of historical threat should show the most punitive current-day religious beliefs, a relationship that should be mediated by states' cultural tightness. Study 5 provides experimental support for this model by manipulating the salience of ecological threat and measuring perceptions of cultural 
tightness, motivation to punish norm violators, and punitive religious belief. Using these data, we fit a full serial mediation from ecological threat $\rightarrow$ support for cultural tightness $\rightarrow$ motivation to punish norm violators $\rightarrow$ punitive religious belief.

We conduct these analyses using data from Christianity in the United States because punitive religious beliefs vary widely both geographically (across states) and historically amongst American Christians. Religious trends within the United States are also welldocumented in large online corpuses of written text and national surveys of religious belief. All of our archival analyses control for general levels of religiosity in order to focus on the specific relationship between cultural tightness and punitive religious beliefs.

\section{Study 1: Cultural Tightness and Historical Change in Punitive Religious Beliefs}

Study 1 explored whether cultural tightness could explain changes in American Christianity from 1800 to 2000 using historical linguistic data from the Google Books American Corpus (GBAC). The GBAC is a 155 billion-word corpus containing a diverse set of written material published in the United States from 1800 onward, and we collected data from this corpus by scraping the "n-gram viewer" at https://books.google.com/ngrams. We only took data from 1800-2000 because the increase in online publications after 2000 makes $21^{\text {st }}$ century data very different in content and potentially unrepresentative of the population. For example, $21^{\text {st }}$ century corpuses are saturated with scientific jargon due to the recent movement towards online scientific journals (Pechenick, Danforth, \& Dodds, 2015).

\section{Method}

Open Science Statement: For all studies, all data were collected prior to analyses, and we report all conditions, measures, and participants. All experimental studies included random 
assignment and the exact language in our manipulations is given in our paper's Appendices. For archival studies we report all measures included in our analyses.

We did not pre-register Study 1 because we used measures that we have already collected and compiled for other analyses (Caluori et al., 2020; Jackson et al., 2019a). However, our code and data are openly available at https://osf.io/hrdje/?view_only=1f0940b6a76246af87cb3e0f4b615643 and we used the same analytic procedures as Caluori and colleagues (2020), who tested for the relationship between warfare and Bible Chapter citations.

Measurement of Punitive Religious Beliefs. The GBAC allows us to assess the frequency of "n-grams" — words or strings of words - across time — a frequency measure that controls for the total number of words published in a year. The n-gram frequencies we assessed were in-text references to 20 Old Testament Bible chapters, 10 which depict God as punitive, and 10 which do not depict God as punitive. These 10 non-punitive chapters allowed us to control for general references to the Bible (which we call "general religiosity"), which likely covaried with punitive religious beliefs. We used the titles of these Bible chapters as search strings ("Job 4") rather than words such as "smite" and "idolatry" in order to avoid confounding changes in the popularity of Biblical words with changes in the popularity of Bible passages themselves.

We first developed this set of Bible chapters to test for the association between warfare and punitive religious beliefs, and the process of selecting and validating these Bible chapters is described in Caluori et al. (2020). In particular, we ensured that "neutral" chapters did not feature any instances of supernatural punishment in order to ensure the face validity of our sample. Punitive chapters include Ezekiel 7, which describes God's punishment of sinners: "Soon now I 
will pour out my wrath upon you; I will spend my anger against you. I will judge you according to your ways, and punish you for all your abominations," and Psalm 78, which describes how God punished those who doubted him despite his generosity, stating "In spite of all this they still sinned; they did not believe in his wonders. So he made their days vanish like a breath, and their years in terror" Non-punitive chapters include Genesis 1, which describes God's actions on the six days of creation and the Sabbath, and Ecclesiastes 3, which puts forth that God has created a time for everything, from birth and death to mourning and rejoicing. Table 1 gives the full set of chapter strings that we used in our analyses, and our supplemental materials (Table S1) provide more information about the contents of each chapter in our analysis.

\begin{tabular}{ll}
\hline Table 1. Punitive and Neutral Bible Chapters in Study 1 \\
\hline Punitive Chapters & Non-Punitive Chapters \\
\hline Job 4 & Ecclesiastes 1 \\
Isaiah 13 & Ecclesiastes 3 \\
Habakkuk 3 & Genesis 11 \\
Exodus 15 & Genesis 1 \\
Ezekiel 7 & Genesis 2 \\
Lamentations 2 & Joshua 1 \\
Psalms 78 & Proverbs 27 \\
Jeremiah 7 & Proverbs 4 \\
Deuteronomy 9 & Leviticus 19 \\
Numbers 11 & Psalms 127 \\
\hline Note. We used cross-year prevalence in these word strings (“Jeremiah 7’) to
\end{tabular}


measure the prevalence of punitive and non-punitive chapter citations.

Measurement of Cultural Tightness. We measured cultural tightness through the frequency of use of the 40 words comprising the tightness-looseness dictionary published by Jackson and colleagues (2019a). Twenty of the words in this dictionary are "tight" because they connote constraint or rule enforcement ("restrain," "comply," "uniformity," and "enforce"). The other twenty words are "loose" because they connote freedom or norm violation ("allow," “freedom," "create," and "openness"). Jackson and colleagues (2019a) recommend subtracting usage of loose words from usage of tight words to create an overall linguistic index of cultural tightness. Tight and loose words were negatively correlated in their usage over time in the GBAC, $\tau=-.62, p<.001$, supporting the validity of a composite index. Jackson and colleagues (2019a) also validated this index by showing that it correlated over time with several other forms of cultural tightness such as number of laws passed and profanities in film and television (reverse scored). We standardized all variables prior to analyses for ease of interpretation.

\section{Results}

Detrending and Stationarity Checks. Before testing our hypotheses, we detrended our time series data to make sure that our variables were not confounded with an underlying trend. Often two time series will correlate, suggesting a meaningful relationship, but the correlation will only result from a shared monotonic trend (e.g. both variables are decreasing over time). To avoid this problem, it is common practice to "detrend" data by removing underlying trends that could produce confounding covariance. We used regression-based residuals to remove variance associated with the monotonic effect of time, and we also removed variance associated with neutral Bible chapters in order to make sure our effects were not confounded with changes to 
religiosity in general. We then tested whether our data were "stationary" (not associated with an underlying trend) using an augmented Dickey-Fuller root test, in which significant values indicate stationarity. Results indicated that both the punitive religious beliefs $(p<.001)$ and cultural tightness $(p=.05)$ time series were stationary, though cultural tightness bordered on marginal significance.

Finally, we plotted the detrended punitive religious beliefs and tightness time series in order to visually assess their relationship. This plot, shown in Figure 2, demonstrated coherence between tightness and punitive religious beliefs. Both time series appeared to rise and fall together, and in many cases, increases in cultural tightness preceded increases in punitive religious beliefs. We next tested for this covariation and lagged influence with a set of regression, cross-correlation, and granger causality models.

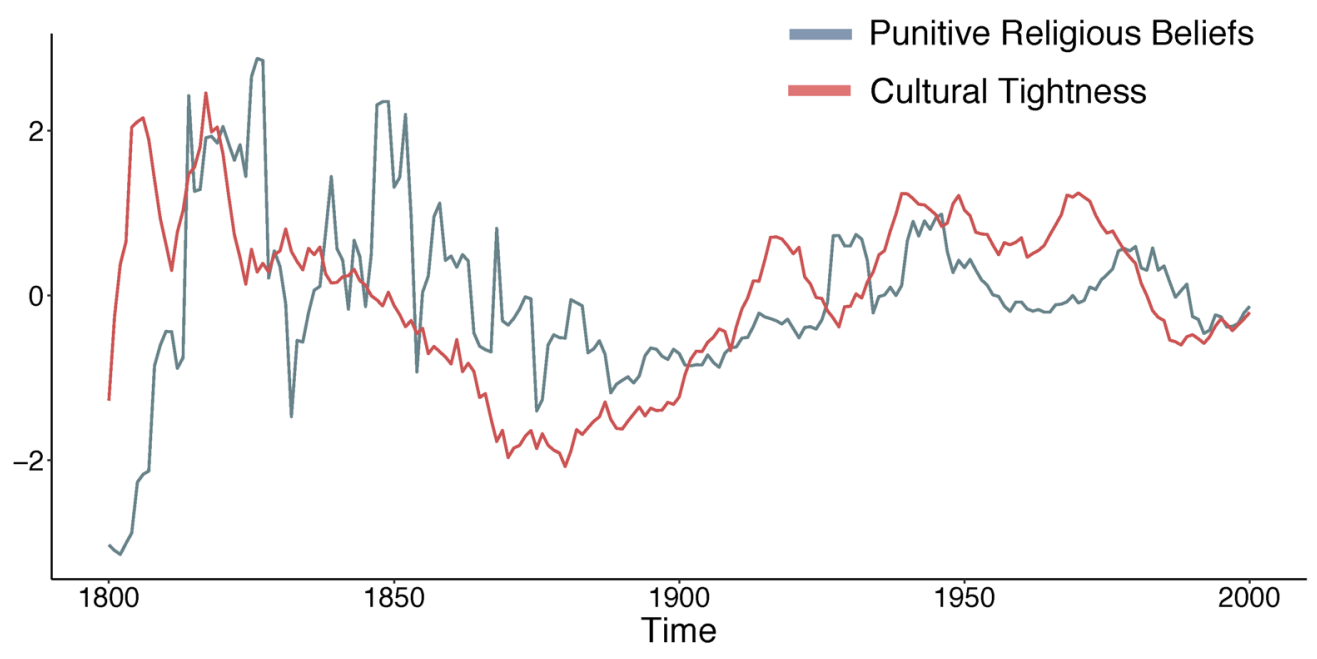

Figure 2. Fluctuations in linguistic markers of cultural tightness and punitive religious beliefs from 1800 to 2000. Data have been detrended and variance in general levels of religiosity has been removed prior to plotting. Each variable has been standardized. 
Regression Analysis. We first tested for the historical relationship between cultural tightness and punitive religious beliefs using regression analysis. Regression analysis can establish correlation but not causality. As predicted, cultural tightness significantly correlated with punitive religious beliefs, $b=.001, \beta=.26, S E=.0004, t(199)=3.76, p<.001$. Since this relationship controlled for general religiosity and year, our link was not confounded with these other variables.

Cross-Correlation Analysis. We next examined whether fluctuations in cultural tightness preceded — rather than just co-occurred with - punitive religious beliefs. We tested this prediction using cross-correlational analysis between cultural tightness and punitive religious beliefs at 60 different lagged intervals.

We "pre-whitened" our time series prior to this analysis. Pre-whitening is helpful for fitting and removing autoregressive processes that may confound a bivariate relationship. Prewhitening is typically considered as an alternative to the detrending that we conducted during preprocessing. However, to leverage a conservative test of our hypothesis, we applied prewhitening in addition to detrending. Pre-whitening's first step takes a bivariate relationship, determines a time series model for variable-x, and stores the model's residuals. Step 2 then filters the time series for variable-y using the variable-x model and determines the differences between the observed values and the "estimated" values using Step 1's fitted model. Finally, Step 3 examines the cross-correlation function between the residuals from Step 1 and the filtered yvalues from Step 2 in order to assess the relationship between $\mathrm{x}$ and $\mathrm{y}$ above and beyond the time series models that could characterize both variables. There is no risk to pre-whitening a time series that has already been detrended, and our results replicated when we detrended without prewhitening. 
The results of our detrended and pre-whitened cross-correlation function are shown in Figure 3. This plot's x-axis represents the lag of a correlation between cultural tightness and punitive religious beliefs, whereas the y-axis represents the strength of the correlation at that lag. For example, if a bar has a value of .30 where the $\mathrm{x}$-axis $=-3$, this means that cultural tightness at time $t$ has a .30 correlation with punitive religious beliefs at time $t$-3 (i.e. punitive religious is preceding tightness), whereas if a bar has a value of .30 where the $\mathrm{x}$-axis $=3$, cultural tightness at time $t$ has a .30 correlation with punitive religious beliefs at time $t+3$ (i.e. cultural tightness is preceding punitive religious beliefs).

Punitive God Predicting CT CT Predicting Punitive God

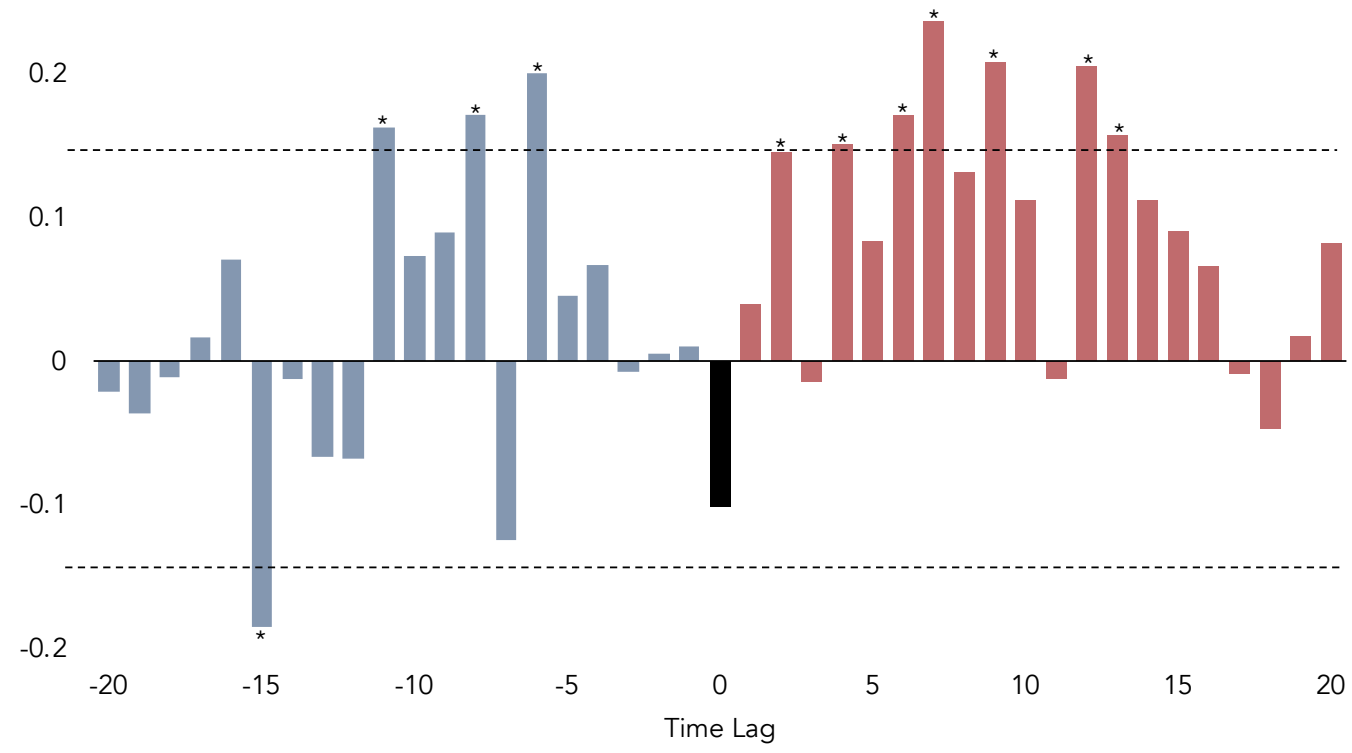

Figure 3. A cross-correlation plot showing the relationship between cultural tightness ("CTL") and punitive religious beliefs ("PRB”). Bars in this plot represent individual Pearson correlations, with the y-value representing the size of the correlation ( $r$ value) and the dashed lines representing the threshold for significant correlations. Negative values on the x-axis (in 
blue) indicate that changes in punitive religious beliefs precede changes in cultural tightness, whereas positive values (in red) indicate that changes in cultural tightness precede changes in punitive religious beliefs. Starred bars are significant correlations.

Consistent with our prediction, cross-correlations indicated a significant positive lag: increases in cultural tightness preceded increases in punitive religious beliefs, with significant lagged effects at $t+2(r=.15), t+4(r=.15), t+6(r=.17), t+7(r=.24), t+9(r=.21), t+12(r=$ $.21)$, and $t+13(r=.16)$. There were also negatively-lagged effects showing that punitive religious beliefs in turn preceded increases in cultural tightness, with significant lags at $t-6(r=$ $.20), t-8(r=.17)$, and $t-11(r=.16)$. These results suggest that cultural tightness and supernatural punishment could be mutually reinforcing: punitive religious beliefs may increase the favorability of abidance to communal norms, and abidance to communal norms may in turn increase punitive religious beliefs.

Granger Causality. Finally, we examined the likelihood that fluctuations in cultural tightness had a pseudo-causal relationship with punitive religious beliefs across time. We examined this prediction using a test of Granger causality, which tested whether our predictor of interest (cultural tightness) "Granger-caused" our outcome of interest (punitive religious beliefs). Granger causality tests whether an endogenous variable (tightness) can predict future changes in an exogenous variable (punitive religious beliefs) above and beyond earlier values of that exogenous variable (Ding, Chen, \& Bressler, 2006). Although Granger causality does not offer the same confidence in causality as an experiment, it does offer stronger causal inference than correlation or regression. Granger tests require specified lagged values, and since we had no 
theoretical priors for how long it takes cultural tightness to translate to punitive religious beliefs, we replicated the model across 5 lagged intervals: 2 years, 4 years, 6 years, 8 years, and 10 years. Consistent with our prediction of temporal causality, Table 2 shows that cultural tightness Granger-caused punitive religious beliefs at each of the lagged intervals, with the strongest effect sizes at shorter lags. Since our data in these tests were detrended and residualized using general religiosity, our significant values cannot be reduced to variation in monotonic autocorrelation or confounding covariance with changes in general levels of religiosity. Since there was some evidence of mutual causality in our cross-correlations, we also tested for reverse Granger causality. In these tests, we found some evidence that punitive religious beliefs Granger-caused tightness, especially at longer intervals.

Table 2. Granger Causality Test Results in Study 1

\begin{tabular}{lcc}
\hline Lag & Tightness $\rightarrow$ Punitive Religious Beliefs & Punitive Religious Beliefs $\rightarrow$ Tightness \\
\hline 2 years & $F(1,196)=5.74, p=.004$ & $F(1,196)=3.25, p=.04$ \\
6 years & $F(1,192)=6.78, p<.001$ & $F(1,192)=-.55, p=.70$ \\
6 years & $F(1,188)=5.54, p<.001$ & $F(1,188)=-.68, p=.66$ \\
8 years & $F(1,184)=5.26, p<.001$ & $F(1,184)=5.06, p<.001$ \\
10 years & $F(1,180)=3.96, p=.001$ & $F(1,180)=3.89, p<.001$ \\
\hline
\end{tabular}

\section{Discussion}

Study 1 supported our hypothesis that cultural tightness predicts and precedes increases in punitive religious beliefs. Linguistic indicators of cultural tightness across 200 years both correlated with and preceded the prevalence of punitive religious beliefs. Moreover, Granger 
causality analyses suggest that temporal fluctuations in punitive religious beliefs over time are caused by preceding fluctuations in cultural tightness, but also suggest the relationship may be mutually causative. This conclusion fits with cultural evolutionary explanations of religion that suggest that punitive beliefs encourage coordination and cooperation (Norenzayan et al., 2016) key facets of tightness. We next tested whether the pseudo-causal relationship between cultural tightness and punitive religious beliefs replicated in a fully causal experimental design.

Study 2: Testing the Causal Link Between Cultural Tightness and Punitive Religious Belief

Study 2 built on Study 1's historical analysis by experimentally testing whether manipulating support for cultural tightness at the individual level increased people's punitive religious beliefs via the importance that people ascribed to punitive traits of God. This experimental design also allowed us to test for the mechanism through which cultural tightness influences religious beliefs. We predicted that motivation to punish norm violators would mediate the relationship between cultural tightness and punitive religious belief.

\section{Method}

Pre-Registration. We pre-registered our hypotheses and study characteristics at https://osf.io/hrdje/?view_only=1f0940b6a76246af87cb3e0f4b615643.

Participants. We recruited 1,000 participants (381 men, 617 women, 2 other; $\left.M_{\text {age }}=49.67, S D_{\text {age }}=17.40\right)$ from across the United States. We used the Qualtrics Panels service to recruit a sample who self-identified as religious, and who were nationally representative in terms of political party affiliation (40\% Democrat, 40\% Republican, 10\% Independent, 10\% “Other”), ethnicity (66\% White, 12\% Black, 12\% Hispanic, 10\% “Other”), region of the country (20\% Midwest, 20\% Northeast, 40\% South, 20\% West), and annual income (35\% below 50k, 35\% 50- 
$100 \mathrm{k}, 30 \%$ over $100 \mathrm{k}$ ). This sample size gave us $88 \%$ power to detect a small effect size of $d=$ .20 .

Manipulation. At the beginning of the study, participants were randomly assigned to a tight condition $(n=492)$ or a loose condition $(n=508)$. In the tight condition, participants read a short paragraph attributing the success of the United States to its strong foundation of law and order. In the loose condition, participants read an identical paragraph, this time attributing the success of the United States to its commitment to freedom and openness. Although manipulating culture-level variables like tightness is difficult in an experimental setting, this approach allowed us to temporarily shift participants' perceptions of their current society. Exact text from both vignettes is available in Appendix A.

To increase the impact of the manipulation, participants in the tight condition were able to personally endorse up to three elements of current American society that "preserve law and order," whereas participants in the loose condition were able to endorse up to three elements of American society that "preserve freedom and openness." Participants were required to endorse at least one of these elements (see Appendix A). We added these endorsement items to increase the strength of our manipulation, since research on cognitive dissonance and self-perception shows that personal endorsement increases the efficacy of persuasion (Bem, 1972; Festinger, 1962).

\section{Measures.}

Motivation to punish norm violators. To assess people's motivation to punish norm violators, we created a measure in which participants rated the extent to which they found each of a series of 5 statements endorsing punishment for norm violators in different contexts (e.g. "It would be appropriate for people who break the law to be harshly punished for their actions," "It would be appropriate for people to report law-breakers to the police") to be appropriate on a 
scale from 1 (highly inappropriate) to 7 (highly appropriate). An exploratory factor analysis with varimax rotation revealed a one-factor solution for this measure, with one factor with an Eigenvalue greater than 1 (Eigenvalue $=2.43$ ) which explained $33 \%$ of the variance and no other factors with Eigenvalues greater than 1. An analysis of these items' internal consistency revealed moderate reliability of $\alpha=.73$, and reliability decreased when any single item was removed from the index. We therefore averaged all items into a single "motivation to punish norm violators" measure. The full set of items is available in our supplemental materials.

Punitive religious importance. Participants rated the extent to which they viewed 9 punitive (Controlling, Restricting, Stern, Commanding, Strict, Angry, Judging, Punishing, Wrathful) and 9 loving (Helping, Generous, Compassionate, Gracious, Tolerant, Caring, Accepting, Merciful, Forgiving) traits as important characteristics of God for American society (Johnson, Okun, \& Cohen, 2015). Trait ratings were averaged to create two composite scores of punitive $(\alpha=.93)$ and loving $(\alpha=.96)$ religious importance. Our primary hypotheses focus on punitive traits, but we included the scale's loving traits for the sake of comprehensiveness.

Procedure. Participants first completed the manipulation. Then they completed the motivation to punish and punitive religious beliefs scales. Finally, participants completed demographics measures.

\section{Results}

Support for Cultural Tightness and Punitive Religious Beliefs. Did manipulating participants' support for cultural tightness vs. looseness change the traits that they viewed as important in God? Independent-samples t-tests revealed that participants in the tight condition $(M=3.99, S D=1.41)$ rated punitive religious traits as significantly more important than participants in the loose condition $(M=3.71, S D=1.41), t(998)=-3.16, p=.002, d=.20,95 \%$ 
CIs $[.08, .32]$. There was no significant difference in the importance of loving traits across the tight $(M=5.61, S D=1.34)$ and loose $(M=5.65, S D=1.28)$ conditions, $t(998)=.54, p=.59$.

\section{Support for Cultural Tightness and Motivation to Punish Norm Violators. As}

predicted, participants in the tight $(M=5.02, S D=1.08)$ condition showed more motivation to punish norm violators than participants in the loose $(M=4.80, S D=1.03)$ condition, $t(998)=$ $3.30, p=.001, d=.2195 \%$ CIs $[.08, .32]$.

Motivation to Punish Norm Violators and Punitive Religious Beliefs. We next tested whether participants with a higher motivation to punish norm violators also found punitive traits more important. As predicted, the motivation to punish norm violators correlated significantly with people's perceived importance of punitive religious traits, $r=.29, p<.001$ (see Figure 4).

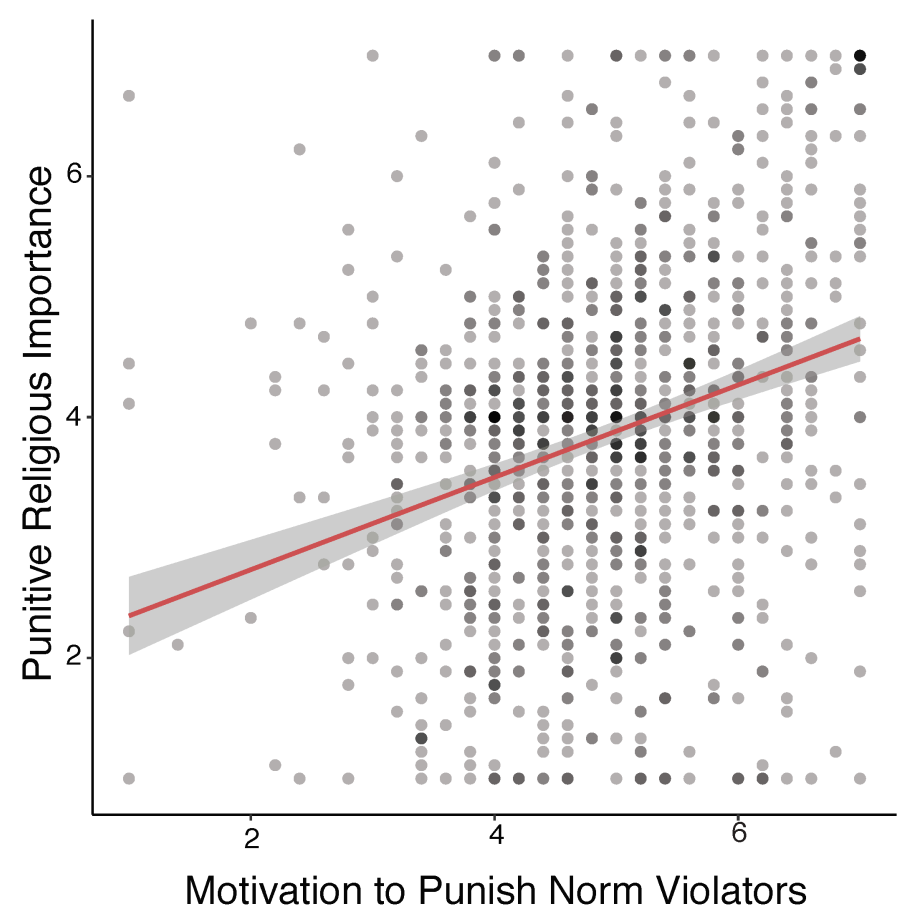

Figure 4. The relationship between motivation to punish norm violators and punitive religious importance in Study 2. Some data points overlap, and data point darkness represents the number of overlapping data points. Shading around the trendline represents standard error. 
Mediation. We predicted that the relationship between our manipulation of support for tightness and punitive religious importance would be mediated by the motivation to punish norm violators. Consistent with this prediction, a 5,000-sample bootstrapped path model found a significant indirect effect of condition on punitive religious importance through the motivation to punish norm violators, $b=.082,95 \%$ CIs $[.030, .13]$. In this model, the direct effect of condition remained significant, $b=.20,95 \%$ CIs $[.03, .37]$, indicating partial mediation. This means that support for tightness most strongly encourages punitive religious importance when it is accompanied by a strong desire to punish rule-breakers, but other factors may also explain the relationship between tightness and punitive religious importance. Figure 5 depicts this mediation.

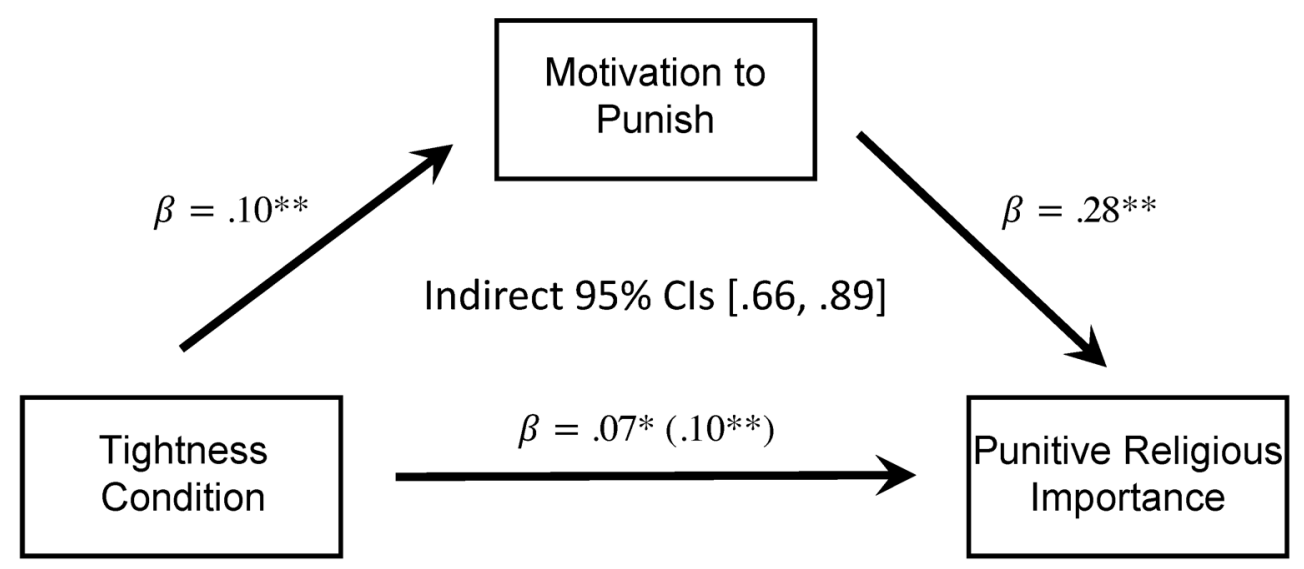

Figure 5. Regression coefficients for the relationship between tightness condition and punitive religious importance as mediated by motivation to punish norm violators in Study 2 . The total effect of tightness condition on punitive religious importance (not controlling for motivation to punish) is in parentheses. Regression coefficients represent standardized estimates.

\section{Discussion}


Study 2 found that support for cultural tightness leads people to find punitive traits more important in God. Manipulating perceptions of cultural tightness resulted in greater endorsement of punitive traits, as well as increased motivation to punish norm violators. Moreover, cultural tightness influenced the perceived importance of a punitive God in part via the motivation to punish norm violators, suggesting that punitive religious beliefs are psychologically attractive for members of tight societies partly because they provide a mechanism through which to punish transgressors (see Laurin, Shariff, Henrich, \& Kay, 2012). We note that the reverse mediational path (Tightness $\rightarrow$ Punitive Religious Importance $\rightarrow$ Motivation to Punish Norm Violators) also reached significance. This suggests that the motivation to punish norm violators and punitive religious beliefs may be mutually enforcing. We document this reverse mediational dynamic in greater detail in our supplemental materials and describe the implications of this effect in our General Discussion.

Nevertheless, Study 2 had three important limitations. First, our manipulation may have increased people's motivation to punish norm violators because it mentioned effective law enforcement, versus increasing people's preference for strong norms. Second, our measure of punitive religious beliefs asked about the "importance" of different traits in God rather than about people's "belief" in these traits. We selected a measure of importance because it is sometimes difficult to manipulate religious belief in an experimental context (Hoogeveen et al., 2019). Nevertheless, people may have endorsed the importance of different traits in our study without necessarily believing them. Finally, we measured the motivation to punish by asking about the appropriateness of punishing norm violators in general. However, people might have interpreted these questions as asking about punishment norms in the United States rather than the 
extent that they were personally motivated to punish norm violators. We addressed each of these limitations in a new experimental study.

\section{Study 3: Replicating the Causal Effect of Cultural Tightness on Punitive Religious Beliefs}

Study 3 replicated the causal relationship between cultural tightness and punitive religious beliefs with a new procedure designed to address the limitations from Study 2. Rather than reading about the benefits of tight vs. loose attributes, participants in Study 3 imagined themselves in a future society, and then answered questions about their personal beliefs and attitudes as a member of this future society. Some participants imagined themselves in a future society with strict norms, whereas other participants imagined themselves in a future society with loose norms. We hypothesized that participants who imagined themselves in a future society with strict norms would express more punitive religious beliefs, and that this effect would be mediated by a greater personal desire to punish norm violators.

\section{Method}

Pilot Study and Pre-Registration. Before pre-registering and running our full study, we conducted an exploratory pilot $(n=99)$ for Study 3 to test if participants' ratings of "important" God traits would align with their ratings of which traits of God they believed in. In this pilot, we found high correlations between measures of importance and belief for punitive $(r=.88)$ and loving $(r=.70)$ attributes of God. Furthermore, our manipulation predicted measures of importance and also belief (see supplemental materials for more details). We chose to adopt a measure explicitly asking participants about their beliefs to show that our effect generalized to a new measure. Our supplemental materials summarize this pilot study in more depth. Following our pilot, we pre-registered our hypotheses and study characteristics at https://osf.io/hrdje/?view_only=1f0940b6a76246af87cb3e0f4b615643. 
Participants. We recruited 2,010 participants from across the United States. 178 participants were excluded from analyses due to a failed attention check, resulting in a final sample of 1,832 participants (447 men, 1,379 women, 6 other; $M_{\mathrm{age}}=34.39, S D_{\mathrm{age}}=16.13$ ). We used the Qualtrics Panels service to recruit a sample who self-identified as Christian and who (as in Study 2) were nationally representative in terms of political party affiliation, race, region of the country, and income. This sample size gave us $99 \%$ power to detect a small effect size of $d=$ .20 .

Manipulation. At the beginning of the study, participants were randomly assigned to a tight condition $(n=955)$ or a loose condition $(n=877)$. Participants in both conditions read a narrative vignette that asked them to imagine that they were a member of a future society-the Tekki - and described a specific custom in which members of this society greet one's family by touching elbows with them. This description was accompanied by a figure that showed two Tekki members participating in this custom (see Kachanoff, Taylor, Caouette, Khullar, \& Wohl, 2019). In the tight condition, participants read that the Tekki have many social norms and strong expectations for members to uphold cultural practices. In the loose condition, participants read that the Tekki have very few social norms and weak expectations for members to uphold them. Although manipulating culture-level variables is difficult in an experimental setting, this approach allowed us to immerse participants in a novel cultural situation and temporarily shift their perceptions of tightness. Exact text from both vignettes is available in Appendix B. After reading the assigned vignette, participants completed two multiple-choice comprehension checks to ensure that they thoroughly read and understood the manipulation. Participants had to answer both checks correctly in order to continue with the rest of the study. For the remaining measures, participants were asked to respond as a member of the Tekki society. 


\section{Measures.}

Motivation to punish norm violators. To assess support for punishment, we used the same scale as in Study 2. As in Study 2, the scale had acceptable reliability $(\alpha=.90)$.

For exploratory purposes, participants also completed two, one-item measures of potential reasons for punishment. Participants indicated the extent to which they agreed with two statements about the primary goal of punishment - one assessing deterrence ("I think that the primary goal of punishment should be to deter future offenses") and one assessing retribution ("I think that the primary goal of punishment should be to even out the wrong that the offender has done")—on a scale from 1 (strongly disagree) to 7 (strongly agree). We summarize effects associated with this measure in the supplemental materials.

Punitive religious beliefs. Participants rated the extent to which they believed that God had each of 18 different punitive and loving traits (e.g. punishing, wrathful, merciful, caring; Johnson, Okun, \& Cohen, 2015) on a scale from 1 (not at all) to 7 (a great deal). Trait ratings were averaged to create two composite scores of punitive $(\alpha=.97)$ and loving $(\alpha=.97)$ religious belief. Our primary hypotheses focus on punitive beliefs, but we included the scale's loving subscale for the sake of comprehensiveness.

Procedure. Participants first completed the manipulation, then completed the motivation to punish and punitive religious beliefs scales in randomized order. Finally, participants completed demographics measures and were debriefed.

\section{Results}

Support for Cultural Tightness and Punitive Religious Beliefs. Did positioning participants in a tight vs. loose imaginary society change how they viewed God? Independentsamples t-tests revealed that participants in the tight condition $(M=4.74, S D=1.52)$ believed 
that God possessed punitive traits significantly more so than participants in the loose condition $(M=1.97, S D=1.17), t(1773.10)=-43.89, p<.001, d=2.03,95 \%$ CIs [-2.89, -2.64$].$ There was also a significant difference in belief in loving traits of God across the tight $(M=4.45, S D=$ $1.77)$ and loose $(M=5.68, S D=1.35)$ conditions, $t(1770.90)=16.83, p<.001, d=.79,95 \%$ CIs $[1.09,1.38]$.

Support for Cultural Tightness and Punishment. Participants in the tight $(M=3.99$, $S D=1.49)$ condition supported punishment more than participants in the loose $(M=2.14, S D=$ 1.33 ) condition, $t(1768.8)=-3.30, p<.001, d=1.39,95 \%$ CIs $[-1.97,-1.73]$.

Punishment and Punitive Religious Beliefs. We next tested whether participants who reported higher support for punishment also expressed more punitive views of God. As predicted, support for punishment correlated significantly with people's belief in punitive God traits, $r=.64, p<.001$ (see Figure 6).

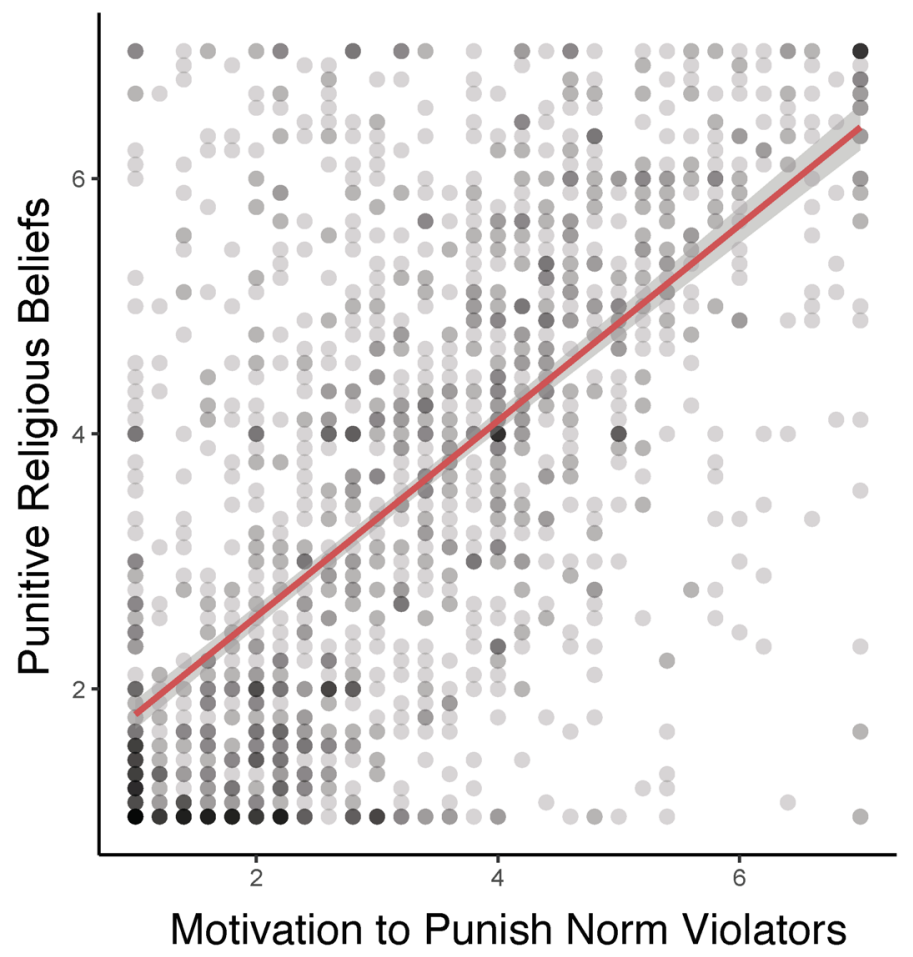


Figure 6. The relationship between motivation to punish norm violators and punitive religious beliefs in Study 3. Some data points overlap, and data point darkness represents the number of overlapping data points. Shading around the trendline represents standard error.

Mediation. We predicted that the relationship between our manipulation of support for tightness and punitive religious beliefs would be mediated by support for punishment. Consistent with this prediction, a 5,000-sample bootstrapped path model found a significant indirect effect of condition on punitive religious beliefs through support for punishment, $b=.77,95 \%$ CIs $[.68$, .87]. In this model, the direct effect of condition remained significant, $b=2.00,95 \% \mathrm{CIs}$ [1.86, 2.14], indicating partial mediation. This means that support for tightness most strongly encourages punitive religious beliefs when it is accompanied by a strong desire to punish rulebreakers, but other factors may also explain the relationship between tightness and punitive religious beliefs. Figure 7 depicts this mediation. As in Study 2, the reverse mediational path (Tightness $\rightarrow$ Punitive God Importance $\rightarrow$ Motivation to Punish Norm Violators) also reached significance, suggesting that the motivation to punish norm violators and punitive religious beliefs may be mutually enforcing. We summarize this reverse mediation in the supplemental materials, and discuss its significance in the General Discussion. 


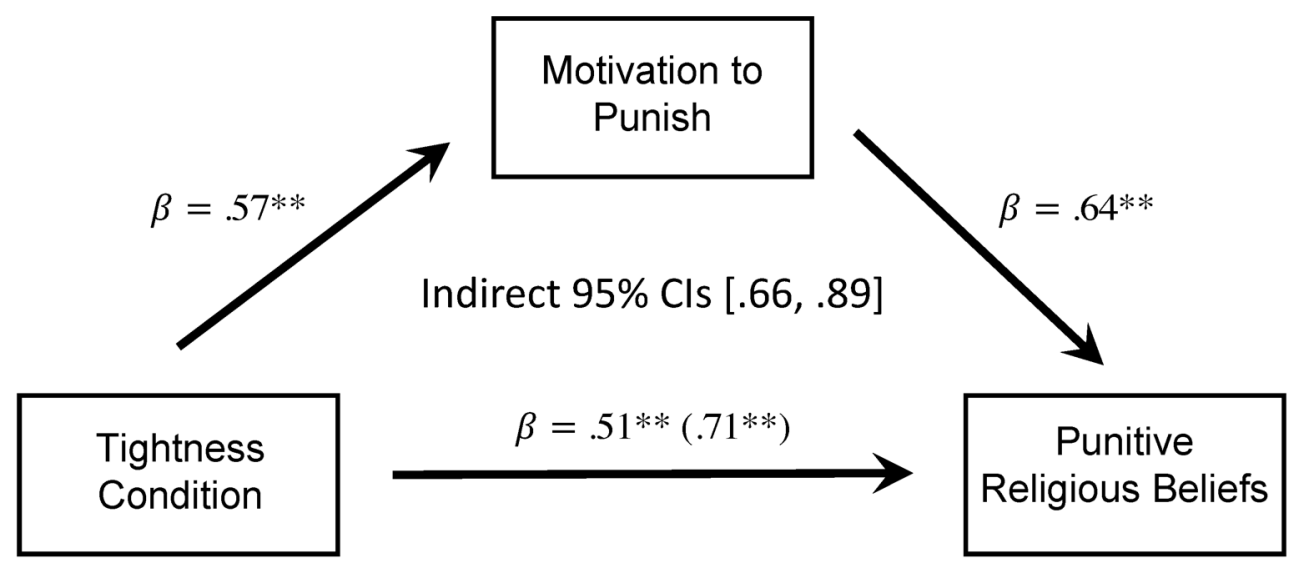

Figure 7. Regression coefficients for the relationship between tightness condition and punitive god belief as mediated by motivation to punish norm violators in Study 3. The total effect of tightness condition on punitive god belief (not controlling for motivation to punish norm violators) is in parentheses. Regression coefficients represent standardized estimates.

\section{Discussion}

Study 3 again found that manipulating cultural tightness increased people's punitive religious beliefs, an effect that was mediated in part by a greater desire to punish. Even with a manipulation which only manipulated the strength of norms (rather than manipulating the strength of norms and also the degree of societal punishment), we found similar effects to Study 2. Having tested the individual-level mechanism by which tightness can encourage punitive religious beliefs, we next turned to examining the society-level dynamics of this relationship and its link with ecological threat.

\section{Study 4: High-Threat States, Cultural Tightness, and Hell Belief}

There is wide diversity in religious beliefs across the United States. For example, the Pew Religious Landscape Survey found that $34 \%$ of Americans in Seattle and 35\% of Americans in Boston believed in Hell—a group-level proxy for punitive religious beliefs (Shariff \& 
Rhemtulla, 2012) — whereas 63\% of Americans in Atlanta and 65\% of Americans in Houston believed in Hell (Pew Research Center, 2014). Study 4 tested whether statewide variation in cultural tightness could predict statewide differences in punitive religious beliefs. Although within-nation variation in religious beliefs is rarely explored, we investigate it here to better reveal why punitive religion might vary meaningfully within a single society.

Regional comparisons of punitive religious beliefs cannot establish that ecological threat or cultural tightness causes punitive religious beliefs, and Study 4 lacked Study 1's longitudinal component ${ }^{1}$. However, our analysis could test whether the relationship between punitive religious belief and ecological threats such as natural disasters, disease, and resource scarcity across regions (see Botero et al., 2014; Gray \& Wegner, 2010; Terrizzi et al., 2012) is mediated by cultural tightness - a key prediction of our model. If cultural tightness mediated the relationship between ecological threats and punitive religious beliefs, this would suggest that ecological threat only predicts greater punitive religious beliefs to the extent that it is also associated with cultural tightness.

We therefore had two central predictions in Study 4. First, we predicted that statewide variation in cultural tightness would correlate with statewide variation in punitive religious beliefs. Second, we predicted that cultural tightness would account for why states with high levels of ecological threat had higher levels of punitive religious beliefs than states with less ecological threat.

\section{Method}

\footnotetext{
${ }^{1}$ The GSS is a multi-wave survey, but our measures of punitive religious beliefs were only included in the 1983-87, 1988-91, 1993-1998, and 2008 waves. Additionally, our measures of cultural tightness and ecological threat were not measured over time, which would prevent longitudinal data analyses such as autoregressive cross-lagged models or analysis of time-varying covariates in latent growth curves. Given these constraints, the only way to conduct an adequately powered and statistically sound analysis of cultural tightness's relationship with punitive religious beliefs was to aggregate across survey waves.
} 
Pre-Registration. We preregistered our hypotheses and analytic plan prior to compiling and analyzing data, and this preregistration is available at https://osf.io/hrdje/?view_only=1f0940b6a76246af87cb3e0f4b615643.

Measurement of Cultural Tightness. Study 4 measured regional variance in statewide cultural tightness with Harrington and Gelfand's (2014) composite index. This index consisted of 9 indicators representing how harshly states punish norm violation (e.g. execution rate from 1976 to 2011, percentage of students hit/punished in schools), reverse-coded latitude/permissiveness (e.g. access to alcohol), and the presence of institutions that enforce order (e.g. state-level religiosity, percentage of people claiming no religious affiliation). In our version of the index, we removed the two religiosity items, in order to avoid confounding tightness with religious belief. Our index correlated highly with the original index, $r=.96, p<.001$.

Measurement of Punitive Religious Beliefs. We measured regional variation in punitive religious beliefs using the American General Social Survey (GSS; $N=52,894)$. Our primary measure assessed belief in Hell's existence with a 4-point Likert- scale with anchors of (1) "Yes, Definitely," (2) "Yes, Probably," (3) "No, Probably Not," (4) "No, Definitely Not." We recoded this question so higher values indicated greater belief in Hell. We also explored the attributes that people assigned to God, but since these items only roughly tapped punitiveness, we present these analyses as exploratory in our paper's supplemental materials. We have uploaded the GSS codebook at https://osf.io/hrdje/?view_only=1f0940b6a76246af87cb3e0f4b615643 so that readers can view the full list of GSS measures.

Measurement of Ecological Threat. We measured statewide ecological threat using a variety of threat indicators from different sources of data, which had previously been compiled into a dataset by Harrington and Gelfand (2014). We used this database to retrieve ecological 
threat variables measuring (a) deaths from a number of natural hazards (heat, cold, flooding, earthquake), (b) tornado risk, (c) food insecurity, (d) poverty rate, (e) parasite stress, and (f) infant mortality rate, which captured a variety of threats facing people in the United States.

Analyses of internal consistency revealed high intercorrelation between these threats $(\alpha=$ .80). States that faced more of one threat were highly likely to face other threats. Therefore, we combined threats into a single "ecological threat" index that summarized the mean level of ecological threat facing each state. Our supplemental materials summarize other approaches, including creating "instability" and "scarcity" factors of ecological threat and modeling each threat separately. Our results were substantively identical in each of these analyses.

Analytic Approach. We tested our hypotheses with a series of regression models. We first tested whether statewide variation in tightness correlated with statewide variation in punitive religious beliefs. Since punitive religious beliefs were measured at the level of the individual, we fit multi-level models with participants nested within states and survey waves, and intercepts randomly varying across states and survey waves when testing for variation in these beliefs.

To test our prediction, we ran four regression models. Model 1 regressed punitive religious beliefs on statewide tightness while controlling for people's general religiosity, which was asked in the GSS with a question where people reported their strength of religious belief on a 4-point scale anchored at 1 (“Strong”) and 4 ("No Religion”). Since our sample identified as Christian, it was not surprising that a minority (11\%) of participants reported "No Religion." This minority may have represented people who were raised Christian but did not consider themselves religious. While we report results with all respondents here, results were similar (and all hypothesized results reached significance) when these "No Religion" participants were excluded from analyses. 
We next tested whether cultural tightness mediated a relationship between statewide variation in ecological threat and variation in punitive religious beliefs. Tightness was measured at the state-level, so we fit general linear models at the state-level when testing for variation in tightness. Models 2-4 first tested the relationship between ecological threat and (a) cultural tightness and (b) punitive religious beliefs. We then conducted a sequence of Monte Carlo simulations — which are effective at probing for indirect effects in multi-level data (Bauer, Preacher, \& Gil, 2006; Selig \& Preacher, 2008) — to test for an indirect effect of ecological threat on punitive religious beliefs through cultural tightness. Our supplemental materials also show that statewide variation in political conservatism — which is highly correlated with religiosity and tightness (Harrington \& Gelfand, 2014)—neither accounts for the link between punitive religious beliefs and cultural tightness nor does it account for the link between punitive religious beliefs and ecological threat.

\section{Results}

Cultural Tightness and Punitive Religious Beliefs. Consistent with our first prediction, statewide tightness positively predicted religious beliefs (measured through beliefs in Hell), even controlling for level of religiosity and conservatism. Religiosity, unsurprisingly, strongly predicted punitive religious beliefs (see Table 3 ).

Ecological Threat, Cultural Tightness, and Punitive Religious Beliefs. Model 2 was a state-level model testing whether ecological threat predicted state-level tightness. Consistent with Harrington and Gelfand (2014), ecological threat predicted tightness (see Table 3).

Models 3-4 next tested whether ecological threat was related to punitive religious beliefs, and whether this relationship was attenuated when modeling the shared variance between tightness and ecological punishment beliefs. Model 3 suggested that ecological threat 
significantly predicts punitive religious beliefs. Model 4 confirmed that, when ecological threat and cultural tightness were modeled together, cultural tightness predicted punitive religious beliefs even controlling for ecological threat. Ecological threat also had a small but significant association with punitive religious beliefs. This is consistent with partial mediation (see Table 3), where a mediator explains some but not all of the relationship between a predictor and an outcome. Figure 8 displays the relationships between ecological threat, cultural tightness, and punitive religious beliefs graphically and in a mediational model.

Table 3. Punitive Religious Beliefs and Cultural Tightness in Study 4

\begin{tabular}{|c|c|c|c|c|c|}
\hline Predictor & Individuals & States & $\beta(\mathrm{SE})$ & $t$ & $p$ \\
\hline Model 1: Hell Beliefs & 3493 & 48 & & & \\
\hline Cultural Tightness & & & $.31(.04)$ & 7.52 & $<.001$ \\
\hline Religiosity & & & $.31(.02)$ & 19.94 & $<.001$ \\
\hline Model 2: Cultural Tightness & & 48 & & & \\
\hline Ecological Threat & & & $.43(.06)$ & 6.99 & $<.001$ \\
\hline Religiosity & & & $.71(.30)$ & 2.34 & .02 \\
\hline Model 3: Hell Beliefs & 3493 & 48 & & & \\
\hline Ecological Threat & & & $.17(.03)$ & 6.78 & $<.001$ \\
\hline Religiosity & & & $.31(.02)$ & 19.99 & $<.001$ \\
\hline Model 4: Hell Beliefs & 3493 & 48 & & & \\
\hline Ecological Threat & & & $.08(.04)$ & 2.34 & .03 \\
\hline Religiosity & & & $.31(.02)$ & 19.92 & $<.001$ \\
\hline Cultural Tightness & & & $.20(.06)$ & 3.35 & .003 \\
\hline
\end{tabular}


Note. Observations vary based on the number individuals who responded to measures. Outcome variable is shown in bold, and predictors are indented below the outcome variable.
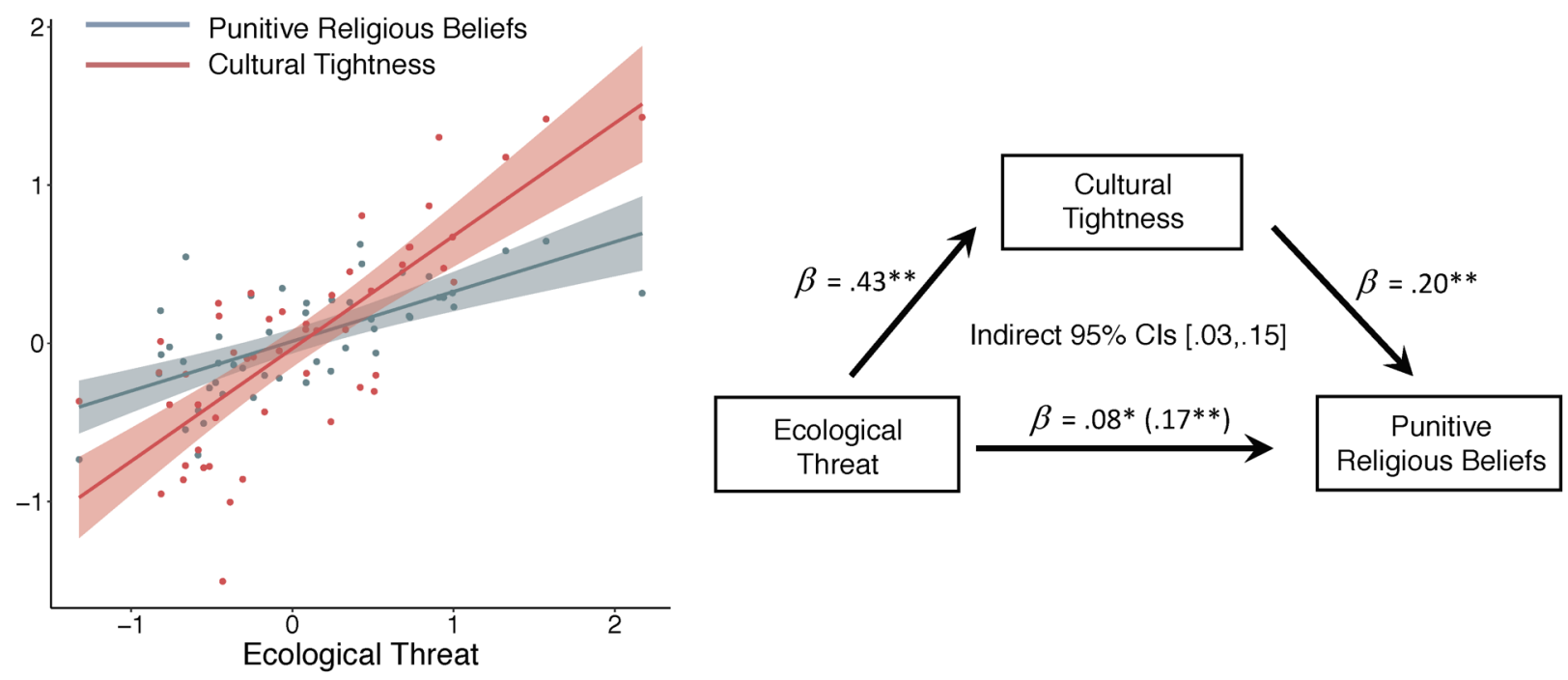

Figure 8. Left: The relationship between ecological threat and (a) statewide tightness (in red) and (b) punitive religious beliefs (in blue). All variables were standardized prior to plotting. Right: A mediation model displaying the relationship between ecological threat and punitive religious beliefs through cultural tightness. Regression coefficients represent standardized estimates. In both models, punitive religious beliefs are measured through Hell beliefs.

Indirect Effects. Our final models formally tested for indirect effects of ecological threat on punitive religious beliefs through cultural tightness in a series of Monte Carlo simulations, using the variance-covariance matrices of models 2-4. These simulations confirmed significant indirect effects of ecological threat on Hell beliefs through cultural tightness, $95 \%$ CIs $[.03,15]$.

\section{Discussion}

This evidence is consistent with our predictions and suggests that cultural tightness at least partially explains why punitive religious beliefs are most common in areas with high levels 
of ecological threat. Moreover, our supplemental materials present a series of models testing for the reverse mediational pathway (ecological threat $\rightarrow$ punitive religious beliefs $\rightarrow$ cultural tightness), showing no evidence for this path.

\section{Study 5: The Causal Link Between Threat and Punitive Belief via Cultural Tightness}

Study 5 aimed to test whether the causal effect of ecological threat on punitive religious beliefs was at least partly mediated by cultural tightness and the motivation to punish norm violators. While this could not capture large-scale cultural dynamics in the same way as Study 4's statewide analysis, it allowed us to test whether dynamic we observed in Study 4 replicates in an experiment—while also adding a measure of people's motivation to punish norm violators in order to capture our complete theoretical model (see Figure 1). We predicted that experimentally increasing the salience of ecological threat would lead people to (a) view cultural tightness as more important for society, (b) show a greater motivation to punish norm violators and (c) endorse punitive religious beliefs. We designed this study by asking people to visualize a future society characterized by either high or low levels of threat. This "future society" design is an emerging paradigm in cultural psychology that has been shown to effectively manipulate perceived cultural context (Caluori et al., 2020).

\section{Method}

Pre-Registration. We pre-registered our hypotheses and study characteristics at https://osf.io/hrdje/?view only=1f0940b6a76246af87cb3e0f4b615643.

Participants. We recruited participants via Amazon Mechanical Turk. We used panels through Cloud Research, a data collection service that works with Mechanical Turk, to ensure that only participants who had previously identified as Christian were invited to complete the survey. We advertised for a sample of 600 Christian participants, as specified in our pre- 
registration, but after excluding people who failed our manipulation checks and failed to complete the study, we were left with a total sample size of 384 participants. Participants were 201 men and 183 women with an average age of 20.25 years $(S D=11.63)$.

Manipulation. At the beginning of the survey, participants were randomly assigned to read a vignette in the societal threat condition $(n=199)$ and a control condition $(n=185)$. In the threat condition, participants were asked to imagine that they lived in a future American society in the year 3000 that faced an increase in natural disasters, disease, and a shortage of food and medical care. In the control condition, participants were asked to imagine that they lived in a future American society that was well-provisioned and had few disasters, diseases, or resource shortages. Since a recent series of studies linked conflict (e.g. warfare) to punitive religious beliefs (Caluori et al., 2020), we omitted conflict from these vignettes to avoid confounding conflict with ecological threat. The full vignettes from the study are listed in Appendix C.

\section{Measures.}

Cultural tightness. We measured participants' perception of cultural tightness with an adapted form of the seven-item scale from past work (Jackson et al., 2019). We used the five items that concerned strictness of norms, in order to avoid confounding support for tight norms with the motivation to punish norm violators. Participants read five incomplete sentences referring to the future society they had read about. They responded to a 1-9 scale in a way that indicated their opinions about how this society would best be organized. For example, one incomplete sentence prompt was "In the face of the conditions described above, it would be important that this future society...," and response options ranged from 1 (never follow the rules) to 9 (be always follow the rules). All prompts were structured so that higher numbers reflected greater support for cultural tightness. This scale was reliable $(\alpha=.83)$. 
Punitive religious beliefs. We measured punitive religious beliefs using the same measure of punitive religious beliefs as in Study 3, rating the extent to which they believed that God had each of 18 different punitive and loving traits (Johnson et al., 2015) on a scale from 1 (not at all) to 7 (a great deal). Trait ratings were averaged to create two composite scores of punitive $(\alpha=.97)$ and loving $(\alpha=.97)$ religious belief. These ratings were averaged to create a composite punitive score $(\alpha=.95)$ and a composite loving score $(\alpha=.95)$. We also measured participants' perceptions of the importance of punitive God traits as in Study 2, but these results were nearly identical to results involving punitive religious beliefs, so we summarize the punitive religious beliefs effects in our main text and present the punitive religious importance analyses in our supplemental materials. As in Studies 2 and 3, our primary hypotheses focus on punitive beliefs, but we included the scale's loving subscale for the sake of comprehensiveness.

Procedure. Participants first completed the manipulation, and then completed the support for cultural tightness measure, the punitive religious beliefs measure, and the motivation to punish norm violators measure in counterbalanced order. They next completed the Supernatural Belief Scale (Jong, Bluemke, \& Halberstadt, 2013), which we only included for potential future exploratory research (it was not included in any preregistered analyses). Participants then completed two manipulation checks to assess whether they had paid attention to and comprehended the manipulation. One of these checks read "The society I read about is facing a high degree of threat" and the other check read "The society I read about is orderly and wellorganized". Participants in the threat condition needed to response "yes" and "no" respectively to be included in analyses, and participants in the control condition needed to respect "no" and “yes" respectively. Results were similar regardless of whether or not we included participants who failed the attention check. Finally, participants completed a demographics questionnaire. 


\section{Results}

Main Effects. As predicted, participants who read about a threatened society endorsed more punitive religious beliefs, greater support for cultural tightness, and a greater motivation to punish norm violators. The means, standard deviations, and t-test statistics for these tests are summarized in Table 4 for the sake of parsimony.

Table 4. All Main Effects of Condition in Study 5

\begin{tabular}{lllll}
\hline Variable & Threat $M(S D)$ & Control $M(S D)$ & $t(\mathrm{DF})$ & $p$ \\
\hline Punitive Religious Belief & $4.08(1.74)$ & $3.31(1.55)$ & $-4.62(381.17)$ & $<.001$ \\
Loving Religious Belief & $5.42(1.46)$ & $6.01(.97)$ & $4.68(346.39)$ & $<.001$ \\
Cultural Tightness & $6.33(1.51)$ & $5.65(1.39)$ & $-4.30(380.86)$ & $<.001$ \\
Motivation to Punish & $4.70(1.31)$ & $4.28(1.31)$ & $-3.12(380.02)$ & .002 \\
\hline
\end{tabular}

Serial Mediation. We predicted that the relationship between societal threat and punitive god importance would be mediated by cultural tightness and the motivation to punish norm violators. We fit this serial mediation as a structural equation model using the lavaan package in R (Rosseel, 2012). Consistent with our prediction, this model's indirect effect reached significance, $b=.10,95 \%$ CIs $[.04, .16]$. We also uncovered several other significant direct effects. For instance, there was a direct association between our threat manipulation and punitive religious belief, and there was a direct association between punitive religious beliefs and cultural tightness. All effects are summarized in Figure 9, which displays the full model. 


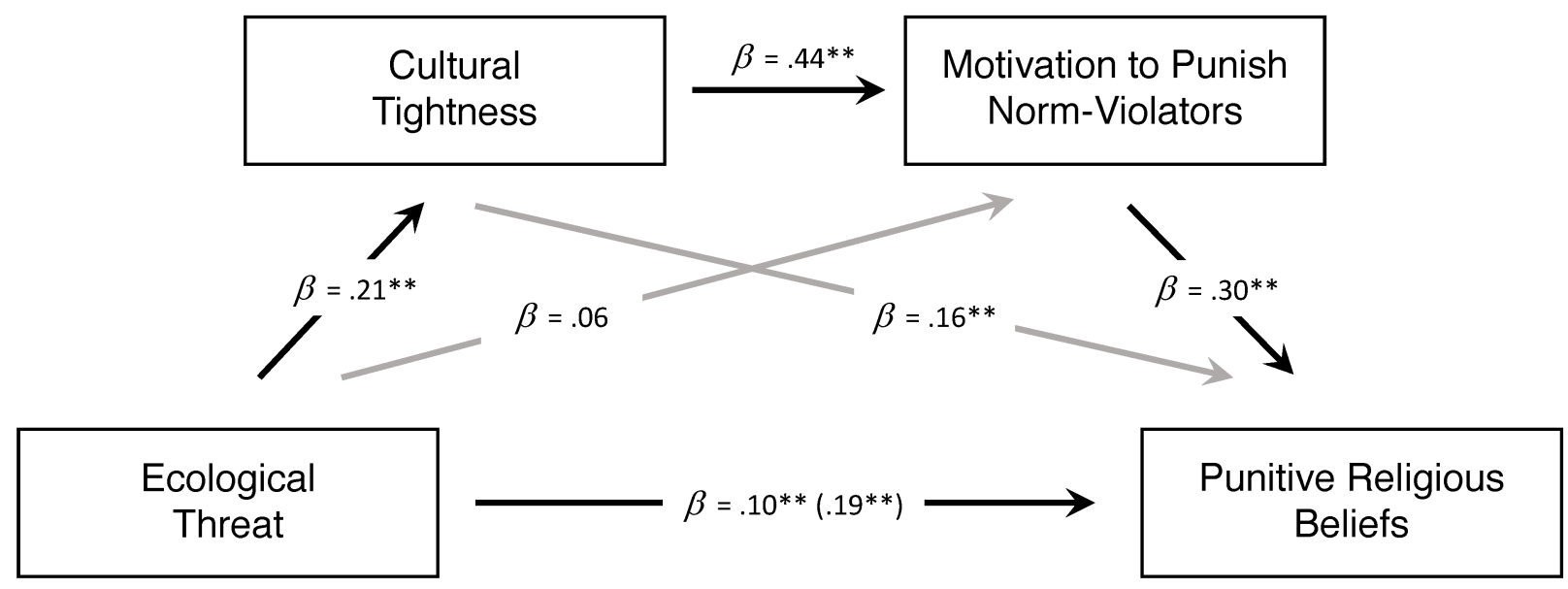

Figure 9. The full serial mediation from ecological threat to punitive religious beliefs through cultural tightness and the motivation to punish norm violators. The total effect of threat on punitive god belief (not controlling for cultural tightness and the motivation to punish norm violators) is in parentheses. Regression coefficients represent standardized estimates.

\section{Discussion}

Study 5 found support for our complete theoretical model: ecological threat increases punitive religious beliefs through cultural tightness and the motivation to punish norm violators. Moreover, our hypothesized indirect effect reached significance in a structural equation model. This study provides causal evidence to the correlational effects we observed in Study 4.

Study 5 also revealed two interesting significant direct effects. First, ecological threat had a significant direct effect on punitive religious beliefs, even after accounting for cultural tightness and the motivation to punish norm violators. This suggests that there are other factors associated with ecological threat (e.g. mood or general negativity) that also drive punitive religious beliefs. Second, there was a direct association between punitive religious beliefs and cultural tightness that was not accounted for by the motivation to punish norm violators. This suggests that punitive religious beliefs have a reciprocal relationship with cultural tightness - a 
dynamic that we observed in Study 1 - although we cannot conclude causality since both variables were measured.

\section{General Discussion}

Moralizing supernatural punishment may represent one of the most significant cultural developments in human history. Indeed, many theories of cultural evolution claim that these punitive religious beliefs helped enable and maintain the growth and complexification of human culture over the last 12,000 years (Johnson, 2016; Norenzayan et al., 2016; Watts et al., 2015). However, there are still many open questions about why people adopt punitive religious beliefs and how these beliefs wax and wane over time. Here we address these questions with a new cultural-psychological model of religion. We claim that cultural tightness catalyzes punitive religious beliefs because it increases people's motivation to punish norm violators, which makes punitive religious beliefs more appealing because punitive religious agents can deal out divine punishment. We also suggest that punitive religious beliefs are more prevalent in contexts with high levels of ecological threat because these environments often encourage cultural tightness.

Our model is supported by five multi-method studies. Our first three studies explored the relationship between cultural tightness and punitive religious beliefs. Study 1 shows that temporal changes in cultural tightness predict and precede fluctuations in punitive religious beliefs from 1800 to $2000 \mathrm{CE}$, and Studies 2-3 experimentally replicated this dynamic and found that the effect of cultural tightness on punitive religious beliefs was partially mediated by a greater motivation to punish norm violators. Our final two studies explored whether cultural tightness could explain the relationship between ecological threat and punitive religious beliefs. Study 4 found that the geographic distribution of punitive religious beliefs closely tracks the geographic distribution of cultural tightness, and that cultural tightness statistically accounts for 
why ecological threats such as disease, natural hazards, and resource scarcity are associated with punitive religious beliefs. Study 5 found that priming the salience of ecological threats increased participants' punitive religious beliefs, and provided support for our full theoretical model. Each of our archival studies (Study 1 and Study 4) controlled for general religiosity and other variables (e.g., conservativism in Study 4, and the monotonic effect of time in Study 1), confirming that tightness is specifically linked to punitive religious beliefs.

\section{Open Questions}

This research program sheds light on how punitive religious beliefs can emerge and spread over time. However, it also raises several provocative open questions about religion and culture.

Why Does Cultural Tightness Catalyze Punitive Religious Beliefs? We suggest that cultural tightness catalyzes punitive religious beliefs primarily because it increases people's motivation to punish norm violators, but this is probably not the only reason that tightness may encourage beliefs in supernatural punishment. One complementary path could be that tight societies have more authoritarian leaders (Gelfand et al., 2011; Jackson et al., 2019b), and people's lay theories of leaders as authoritarian spills over into their religious beliefs. Another compatible possibility is that tight societies are characterized by more threat, and the negativity of these events translates to more negative views of god. Some studies suggest that mood effects are not sufficient by themselves to explain the impact of tightness on religion (Caluori et al., 2020). However, this does not mean that mood does not contribute to religious belief. Indeed, the fact that motivation to punish norm violators showed only partial mediation suggests that there are multiple mechanisms that explain why tightness and ecological threat can produce punitive religious beliefs. 


\section{What is the Causal Direction Between Cultural Tightness and Punitive Religious}

Beliefs? Here we suggest that cultural tightness causes more punitive religious beliefs, but past work suggests that punitive religious beliefs can facilitate social complexity and parochial cooperation, which are both common in tight societies (Watts et al., 2015; Purzycki et al., 2016). This suggests that cultural tightness and punitive religious may be reciprocal — each feeding into the other. Indeed, this possibility is supported by our reciprocal time-series effects in Study 1, the reverse mediation that we observed in Studies 2-3, and the direct association between cultural tightness and punitive religious beliefs that we observed in Study 5. These reciprocal effects suggest that secular and religious culture are closely intertwined.

Will Punitive Beliefs Wax or Wane in the Future? Data from Study 1 shows how punitive religious beliefs have waxed and waned over the past 200 years, but these data also raise the question of how religious beliefs will change in the future. Since the Google NGram corpus only includes data until 2008, we cannot generate testable forecasts of how punitive beliefs may change in the future. However, other survey-based and text-based analyses may be able to generate these forecasts and shed light on the future of religion in America and around the world.

\section{Limitations and Future Directions}

Our findings do not imply that cultural tightness is the only catalyst of punitive religious beliefs, which may sometimes arise randomly through copying errors. For example, one community may borrow another community's folktale but misremember it to include a deity punishing someone for misbehavior (Eerkins \& Lipo, 2005). Punitive religious beliefs may also arise due to sociological factors such as political coercion and incentivization (Kane \& Park, 2009; Lydon, 2009). For example, Watts and colleagues (2019) show how Christianity spread fastest in small, politically structured societies across the Pacific Islands. In these societies, 
missionaries could bribe leaders, who could then disseminate Christian materials throughout a society. Furthermore, future research needs to test the generalizability of our results to other cultures. Although there are many strengths to investigating changes in religion within a single society_especially in the United States, which has rich religious diversity (Jackson, Hester, \& Gray, 2018; Pew Research Center, 2014) — it is an open question of whether cultural tightness catalyzes punitive religious beliefs in all cultures.

Future research could also investigate the relative strength of different socioecological pressures in shaping religious beliefs. Previous work has examined how factors such as climate unpredictability (Botero et al., 2014), intergroup conflict (Caluori et al., 2020; Henrich et al., 2019), and crime (Purzycki, 2013) relate to religious beliefs, and we combine these different threats in our analyses. In our analysis, various forms of ecological threat were equally predictive of punitive beliefs (see supplemental materials), but other work suggests that individual-level perceptions of conflict may motivate punitive religious beliefs more than other forms of threat (Caluori et al., 2020). Future research could test how perceived threats vs. actual threats shape punitive religious beliefs, and whether some threats only increase punitive religious beliefs when they are experienced (rather than just perceived).

\section{Conclusion}

Why do people adopt beliefs in gods that punish them? Whereas past research has shown how the modern emergence of punitive religious beliefs has catalyzed significant cultural changes (Norenzayan et al, 2016; Johnson, 2005), we suggest that cultural changes can also catalyze punitive religious beliefs. Our account adds to recent cultural evolutionary models of the self (Talhelm et al., 2014), cultural sensitivity to norms (Kitayama et al., 2016; Mu et al. 2015), 
and prejudice (Jackson et al., 2019b), and brings us one step closer to understanding the origins and development of paranormal punishment and divine damnation.

\section{Statement of Context and Novelty}

Many of today's religions feature moralizing supernatural punishment, but it is still not clear why people originally adopted these beliefs. Indeed, the origin of punitive religious beliefs has become one of the most hotly debated topics among those who study cultural evolution (Whitehouse et al., 2019; Beheim et al., 2020). This paper is intended to harness insights from tightness-looseness theory (Gelfand, Harrington, \& Jackson, 2017) to explain why people might feel motivated to adopt punitive religious beliefs, and why these beliefs might be most common in areas of the world with high levels of collective threat. In recent work, we showed that people's motivation for stricter societies could explain why punitive religious beliefs increase during times of warfare (Caluori et al., 2020). Here, we offer a more expansive model showing that cultural tightness explains the historical and geographic distribution of punitive religious beliefs, and that people's motivation to punish norm violators serves as the individual-level mechanism that links cultural tightness to punitive religious belief. Our ultimate goal is to link psychological insights into retributive justice and deviance with large-scale patterns of historical and cross-cultural variation in religion. 


\section{References}

Aktas, M., Gelfand, M. J., \& Hanges, P. J. (2016). Cultural tightness-looseness and perceptions of effective leadership. Journal of Cross-Cultural Psychology, 47(2), 294-309.

Bauer, D. J., Preacher, K. J., \& Gil, K. M. (2006). Conceptualizing and testing random indirect effects and moderated mediation in multilevel models: new procedures and recommendations. Psychological Methods, 11(2), 142-163.

Beheim, B., Atkinson, Q., Bulbulia, J., Gervais, W. M., Gray, R., Henrich, J., ... \& Purzycki, B. G. (2020). Corrected analyses show that moralizing gods precede complex societies but serious data concerns remain. Preprint at https://psyarxiv.com/jwa2n/download?format=pdf.

Bem, D. J. (1972). Self-perception theory. In Advances in experimental social psychology (Vol. 6, pp. 1-62). Academic Press.

Billing, J., \& Sherman, P. W. (1998). Antimicrobial functions of spices: why some like it hot. The Quarterly Review of Biology, 73(1), 3-49.

Botero, C. A., Gardner, B., Kirby, K. R., Bulbulia, J., Gavin, M. C., \& Gray, R. D. (2014). The ecology of religious beliefs. Proceedings of the National Academy of Sciences, 111(47), 16784-16789.

Brewer, J., Gelfand, M., Jackson, J. C., MacDonald, I. F., Peregrine, P. N., Richerson, P. J., ... \& Wilson, D. S. (2017). Grand challenges for the study of cultural evolution. Nature Ecology \& Evolution, 1(3), 1-3.

Bulbulia, J. (2004). The cognitive and evolutionary psychology of religion. Biology and Philosophy, 19(5), 655-686.

Caluori, N. C., Jackson, J. C., Gray, K., Gelfand, M. (2020). Conflict changes how people view 
God. Psychological Science. https://doi.org/10.1177/0956797619895286

Chua, R. Y., Roth, Y., \& Lemoine, J. F. (2015). The impact of culture on creativity: How cultural tightness and cultural distance affect global innovation crowdsourcing work. Administrative Science Quarterly, 60(2), 189-227.

Ding, M., Chen, Y., \& Bressler, S. L. (2006). Granger causality: basic theory and application to neuroscience. In Schelter, B., Winterhalder, M., Timmer, J. (Eds.), Handbook of time series analysis: Recent theoretical developments and applications (pp.437-460). WileyVCH Verlag.

Eerkens, J. W., \& Lipo, C. P. (2005). Cultural transmission, copying errors, and the generation of variation in material culture and the archaeological record. Journal of Anthropological Archaeology, 24(4), 316-334.

Festinger, L. (1962). A theory of cognitive dissonance (Vol. 2). Stanford university press.

Fogelin, L. (2007). The archaeology of religious ritual. Annual Review of Anthropology, 36, 5571.

Gelfand, M. J., Raver, J. L., Nishii, L., Leslie, L. M., Lun, J., Lim, B. C., ... \& Aycan, Z. (2011). Differences between tight and loose cultures: A 33-nation study. Science, 332(6033), 1100-1104.

Gelfand, M. J., Harrington, J. R., \& Jackson, J. C. (2017). The strength of social norms across human groups. Perspectives on Psychological Science, 12(5), 800-809.

Gelfand, M., Jackson, J. C., \& Harrington, J. R. (2016). Trump culture: Threat, fear and the tightening of the American mind. Scientific American. Retrieved from https://www.scientificamerican.com/article/trump-culture-threat-fear-and-the-tighteningof-the-american-mind/. 
Gray, K., \& Wegner, D. M. (2010). Blaming God for our pain: Human suffering and the divine mind. Personality and Social Psychology Review, 14(1), 7-16.

Gray, R. D., \& Watts, J. (2017). Cultural macroevolution matters. Proceedings of the National Academy of Sciences, 114(30), 7846-7852.

Grossmann, I., \& Varnum, M. E. (2015). Social structure, infectious diseases, disasters, secularism, and cultural change in America. Psychological Science, 26(3), 311-324.

Hackett, C., Grim, B., Stonawski, M., Skirbekk, V., Potančoková, M., \& Abel, G. (2012). The global religious landscape. Washington, DC: Pew Research Center.

Harrington, J. R., \& Gelfand, M. J. (2014). Tightness-looseness across the 50 united states. Proceedings of the National Academy of Sciences, 111(22), 7990-7995.

Henrich, J., Bauer, M., Cassar, A., Chytilová, J., \& Purzycki, B. G. (2019). War increases religiosity. Nature Human Behaviour, 3(2), 129.

Hoogeveen, S., Wagenmakers, E. J., Kay, A. C., \& van Elk, M. (2019). Compensatory Control and Belief in God: A Registered Replication Report Across Two Countries. Comprehensive Results in Social Psychology. https://doi.org/10.1080/23743603.2019.1684821

Jackson, J. C., Ember, C., \& Gelfand, M. (2020). A global analysis of cultural tightness in nonindustrial societies. Preprint available at https://psyarxiv.com/9s57z/download?format=pdf.

Jackson, J. C., Gelfand, M., De, S., \& Fox, A. (2019a). The loosening of American culture over 200 years is associated with a creativity-order trade-off. Nature Human Behaviour, 3(3), 244.

Jackson, J. C., Hester, N., \& Gray, K. (2018). The faces of God in America: Revealing religious 
diversity across people and politics. PloS one, 13(6), e0198745.

Jackson, J. C., van Egmond, M., Choi, V. K., Ember, C. R., Halberstadt, J., Balanovic, J., ... \& Fulop, M. (2019b). Ecological and cultural factors underlying the global distribution of prejudice. PloS one, 14(9), e0221953.

Johnson, D. D. (2005). God's punishment and public goods. Human Nature, 16(4), 410-446.

Johnson, D. (2016). God is watching you: How the fear of God makes us human. Oxford University Press, USA.

Johnson, D., \& Krüger, O. (2004). The good of wrath: Supernatural punishment and the evolution of cooperation. Political Theology, 5(2), 159-176.

Johnson, K. A., Okun, M. A., \& Cohen, A. B. (2015). The mind of the Lord: Measuring authoritarian and benevolent God representations. Psychology of Religion and Spirituality, 7(3), 227.

Jong, J., Bluemke, M., \& Halberstadt, J. (2013). Fear of death and supernatural beliefs: Developing a new supernatural belief scale to test the relationship. European Journal of Personality, 27(5), 495-506.

Kachanoff, F. J., Taylor, D. M., Caouette, J., Khullar, T. H., \& Wohl, M. J. (2019). The chains on all my people are the chains on me: Restrictions to collective autonomy undermine the personal autonomy and psychological well-being of group members. Journal of Personality and Social Psychology, 116(1), 141-165.

Kane, D., \& Park, J. M. (2009). The puzzle of Korean Christianity: Geopolitical networks and religious conversion in early twentieth-century East Asia. American Journal of Sociology, 115(2), 365-404.

Kitayama, S., King, A., Hsu, M., Liberzon, I., \& Yoon, C. (2016). Dopamine-system genes and 
cultural acquisition: The norm sensitivity hypothesis. Current Opinion in Psychology, 8, 167-174.

Lydon, J. (2009). Fantastic dreaming: The archaeology of an aboriginal mission. Rowman Altamira.

Mesoudi, A., Whiten, A., \& Laland, K. N. (2006). Towards a unified science of cultural evolution. Behavioral and Brain Sciences, 29(4), 329-347.

Mu, Y., Kitayama, S., Han, S., \& Gelfand, M. J. (2015). How culture gets embrained: Cultural differences in event-related potentials of social norm violations. Proceedings of the National Academy of Sciences, 112(50), 15348-15353.

Murdock, G. P., \& Provost, C. (1973). Measurement of cultural complexity. Ethnology, 12(4), 379-392.

Norenzayan, A., \& Shariff, A. F. (2008). The origin and evolution of religious prosociality. Science, 322(5898), 58-62.

Norenzayan, A. (2013). Big gods: How religion transformed cooperation and conflict. Princeton University Press.

Norenzayan, A., Shariff, A. F., Gervais, W. M., Willard, A. K., McNamara, R. A., Slingerland, E., \& Henrich, J. (2016). The cultural evolution of prosocial religions. Behavioral and Brain Sciences, 39.

Laurin, K., Shariff, A. F., Henrich, J., \& Kay, A. C. (2012). Outsourcing punishment to God: Beliefs in divine control reduce earthly punishment. Proceedings of the Royal Society B: Biological Sciences, 279(1741), 3272-3281.

Pechenick, E. A., Danforth, C. M., \& Dodds, P. S. (2015). Characterizing the Google Books 
corpus: Strong limits to inferences of socio-cultural and linguistic evolution. PloS one, 10(10), e0137041.

Peoples, H. C., Duda, P., \& Marlowe, F. W. (2016). Hunter-gatherers and the origins of religion. Human Nature, 27(3), 261-282.

Pelto, P. J. (1968). The differences between" tight" and" loose" societies. Trans-action, 5, 37-40.

Pew Research Center (2014). Pew Religious Landscape Survey. Retrieved from https://www.pewforum.org/about-the-religious-landscape-study/.

Purzycki, B. G. (2013). The minds of gods: A comparative study of supernatural agency. Cognition, 129(1), 163-179.

Purzycki, B. G., Apicella, C., Atkinson, Q. D., Cohen, E., McNamara, R. A., Willard, A. K., ... \& Henrich, J. (2016). Moralistic gods, supernatural punishment and the expansion of human sociality. Nature, 530(7590), 327.

Purzycki, B. G., Stagnaro, M. N., \& Sasaki, J. (2020) Breaches of trust change the content and structure of religious appeals. Preprint available at https://www.researchgate.net/profile/Benjamin_Purzycki/publication/332571420_Breach es_of_Trust_Change the Content_and_Structure_of_Religious_Appeals/links $/ 5$ cbebe 52 4585156cd7ab63d5/Breaches-of-Trust-Change-the-Content-and-Structure-of-ReligiousAppeals.pdf

Roos, P., Gelfand, M., Nau, D., \& Lun, J. (2015). Societal threat and cultural variation in the strength of social norms: An evolutionary basis. Organizational Behavior and Human Decision Processes, 129, 14-23.

Rosseel, Y. (2012). Lavaan: An R package for structural equation modeling and more. Version 0.5-12 (BETA). Journal of Statistical Software, 48(2), 1-36. 
Selig, J. P., \& Preacher, K. J. (2008). Monte Carlo method for assessing mediation: An interactive tool for creating confidence intervals for indirect effects [Computer software].

Slingerland, E., Monroe, M. W., Spicer, R., \& Muthukrishna, M. (2020). Historians respond to Whitehouse et al. (2019) "Complex societies precede moralizing gods throughout world history". Preprint available at https://psyarxiv.com/2amjz.

Shariff, A. F., Willard, A. K., Andersen, T., \& Norenzayan, A. (2016). Religious priming: A meta-analysis with a focus on prosociality. Personality and Social Psychology Review, 20(1), 27-48.

Shariff, A. F., \& Rhemtulla, M. (2012). Divergent effects of beliefs in Heaven and Hell on national crime rates. PloS one, 7(6), e39048.

Sperber, D. (1996). Explaining culture: A naturalistic approach. Cambridge, MA: Cambridge.

Talhelm, T., Zhang, X., Oishi, S., Shimin, C., Duan, D., Lan, X., \& Kitayama, S. (2014). Largescale psychological differences within China explained by rice versus wheat agriculture. Science, 344(6184), 603-608.

Terrizzi Jr, J. A., Shook, N. J., \& Ventis, W. L. (2012). Religious conservatism: An evolutionarily evoked disease-avoidance strategy. Religion, Brain \& Behavior, 2(2), 105 120.

Tinbergen, N. (1963). On aims and methods of ethology. Zeitschrift für tierpsychologie, 20(4), 410-433.

Triandis, H. C. (1989). The self and social behavior in differing cultural contexts. Psychological Review, 96(3), 506-520.

Varnum, M. E., \& Grossmann, I. (2017). Pathogen prevalence is associated with cultural changes in gender equality. Nature Human Behaviour, 1(1), 0003. 
Watts, J., Greenhill, S. J., Atkinson, Q. D., Currie, T. E., Bulbulia, J., \& Gray, R. D. (2015). Broad supernatural punishment but not moralizing high gods precede the evolution of political complexity in Austronesia. Proceedings of the Royal Society B: Biological Sciences, 282(1804), 2014-2556.

Watts, J., Sheehan, O., Bulbulia, J., Gray, R. D., \& Atkinson, Q. D. (2018). Christianity spread faster in small, politically structured societies. Nature Human Behaviour, 2(8), 559.

White, C. J., Kelly, J. M., Shariff, A. F., \& Norenzayan, A. (2020). Supernatural norm enforcement: Thinking about Karma and God reduces selfishness among believers. Journal of Experimental Social Psychology. https://doi.org/10.1016/j.jesp.2019.03.008

Whitehouse, H., François, P., Savage, P. E., Currie, T. E., Feeney, K. C., Cioni, E., ... \& ter Haar, B. (2019). Complex societies precede moralizing gods throughout world history. Nature, 568(7751), 226.

Yilmaz, O., \& Bahçekapili, H. G. (2016). Supernatural and secular monitors promote human cooperation only if they remind of punishment. Evolution and Human Behavior, 37(1), 79-84. 


\section{Acknowledgements}

This research was supported by a National Science Foundation Graduate Research Fellowship and a Thomas S. and Caroline H. Royster Fellowship to the first author. This research was also supported by a John Templeton Grant (no. 61111) to the first author and the senior author, who is also supported by funding from the Charles Koch Foundation. No funding agency was involved in the conceptualization, design, data collection, analysis, decision to publish, or preparation of this manuscript, and the views expressed in this manuscript do not necessarily reflect the views of our funding agencies.

\section{Author Contributions}

JCJ conceptualized and designed the study. JCJ, SA, NC, and EB acquired and analyzed the data. JCJ, NC, and SA interpreted the analysis. JCJ wrote the manuscript. KG, NC, SA, and MG revised the manuscript. All authors approved the submitted manuscript.

\section{Competing interests}

The authors declare no competing interests 


\section{Appendix A: Primes from Study 2}

Tight Condition: Most historians believe that the United States was so successful because it was built on a foundation of law and order. From its inception, the United States was effectively able to prevent crime through a large organized police system, and prevent the spread of disease through effective travel and quarantining.

Loose Condition: Many historians believe that the United States was so successful because it was built on a foundation of freedom and openness. From its inception, the United States was effectively able to prevent oppression through free speech for all, and prevent persecution through diversity and inclusive values 


\section{Appendix B: Primes from Study 3}

Tight Condition: Imagine that you are a member of the Tekki, a future society that comes into existence around 500 years from now. You have lived with the Tekki your entire life, and you consider yourself a citizen of Tekki culture with knowledge of and respect for your group's customs. The Tekki have many social norms, and there are strong expectations for members to uphold these practices. For example, it is customary to greet one's family members by touching your elbows to theirs (see picture below).

Most everyone follows this cultural practice, and people would be stunned if any member of the Tekki failed to participate in this custom. You remember hearing stories about a Tekki who once tried to tap shoulders with someone outside of his family; the rest of the Tekki were shocked. "We've always done things the same way," someone told you. "Everyone is expected to follow all of our rules and customs." As a member of the Tekki, you actively participate in this family-elbow-touching practice and all other social norms.

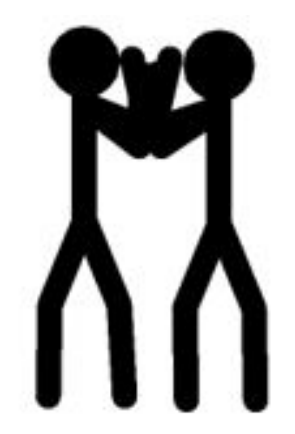

Loose Condition: Imagine that you are a member of the Tekki, a future society that comes into existence around 500 years from now. You have lived with the Tekki your entire life, and you consider yourself a citizen of Tekki culture with knowledge of and respect for your group's customs. The Tekki have very few social norms, and there are little to no expectations for 
members to uphold these practices. For example, it is customary to greet one's family members by touching your elbows to theirs (see picture below).

Some people follow this cultural practice and some don't, and it wouldn't be considered out of the ordinary if any member of the Tekki failed to participate in this custom. Some Tekki tap shoulders with people outside of their family, but no one finds this strange or surprising. "We've always allowed people to do things differently," someone told you. "No one really cares if someone doesn't follow our rules and customs." As a member of the Tekki, you participate in some (but not all) social norms.

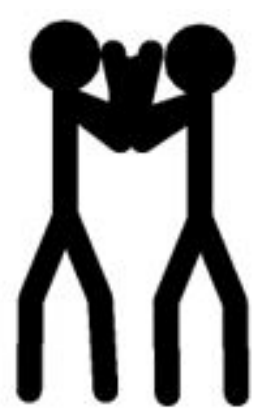




\section{Appendix C: Primes from Study 5}

Threat Condition: Imagine that you are living in the United States in the year 3000 . The frequency of natural disasters (such as earthquakes, tornadoes, forest fires, flooding) has increased dramatically. Food scarcity has also affected a large portion of the nation. The frequency of diseases and epidemics has increased, and adequate medical care has become more and more difficult to access. Society is becoming increasing chaotic, and existing infrastructure cannot provide aid to all who need it.

Control Condition: Imagine that you are living in the United States in the year 3000 . The nation has been peaceful and calm for many years, and new advances in agriculture, climate, and medical research mean that diseases and natural disasters are increasingly rare, and everyone is well-fed. Existing infrastructure has been able to provide aid to all who need it. People feel safe and secure, and there is no sign that this will change in the future. 